\title{
Piezomagnetic Field Associated with the Mogi Model Revisited: Analytic Solution for Finite Spherical Source
}

\author{
Yoichi SASAI \\ Earthquake Research Institute, University of Tokyo, Bunkyo-ku, Tokyo 113, Japan
}

(Received August 1, 1990; Revised October 9, 1990)

\begin{abstract}
The piezomagnetic field associated with the Mogi model was reexamined for the point source and the finite spherical source problems. In the point source case the question was how to deal with divergent stresses near the pressure source. In SASAI's (1979) method, the magnetized crust was divided into two layers, i.e. the upper half shallower than the source $(0<z<D)$ and the lower half deeper than the source $(D<z<H)$. The solution (type I) was simply the sum of the two contributions as calculated by the Fourier transform method. An alternative calculation (type II) integrates the whole magnetized area except for an infinitesimally small sphere involving the pressure source. This was achieved with the aid of a theorem for spherical harmonics. These two procedures gave different results. In order to identify the cause of the discrepancy, the finite source problem was further investigated. The piezomagnetic field was represented in the form of a one-dimensional integral containing complete elliptic integrals. It can be evaluated numerically with the aid of the double exponential formula (DEF). We called it the type III solution. The present analytic approach verifies the results of numerical 3-D integrations by DAVIS (1976), and SUZUKI and OshimAN (1990). The solution as the spherical radius diminishes to zero coincides with the type II solution. The type II solution had a gap across $H=D$. The potential value at $H=D$ was just the average of the two limiting values for $H=D-\varepsilon$ and $H=D+\varepsilon$. In the type I solution, the magnetization along a thin layer at $H=D$ was ignored, which produced the pressure source term in the type II solution. Accordingly the type II solution was appropriate for the point source problem: the previous result by SASAI (1979) should be rejected.
\end{abstract}

\section{Introduction}

A hydrostatically-pumped sphere buried within a semi-infinite elastic medium is called the Mogi model in volcanology. The source sphere is an analogue to a magma reservoir. This mechanical model proposed by MOGI (1958) has successfully explained surface deformations associated with volcanic eruptions (e.g. FISKE and KINOSHITA, 1969). Inflation of a magma chamber produces stresses in the surrounding magnetoelastic medium and hence the stress-induced magnetization. In volcanoes we may expect stress-induced magnetic changes, in addition to those due to thermal demagnetization (STACEY et al., 1965).

The Mogi model has been best investigated in recent studies of tectonomagnetism. DAVIS (1976) numerically calculated piezomagnetic changes associated with the Mogi model in reference to the Kilauea Volcano. It was the first example of the piezomagnetic model in three dimensions, which broke a path to various fault models (e.g. JoHNSTON, 1978; OHSHIMAN, 1980). High-speed computers enabled us to make such elaborate 
numerical integrations. SASAI (1979) obtained an analytical solution of the problem for a point source case. In spite of a rather complicated mathematical treatment, the final result was quite simple: the piezomagnetic field caused by the Mogi model can be represented by magnetic dipoles at the source position as well as dipoles and quadrupoles at two image points with respect to the Curie depth.

The Mogi model is typical for those of tectonic problems. We can easily calculate surface displacements and expansion of the pressure source. These are nothing but the deformation of magnetic substances, so that they also produce magnetic changes. Comparing such changes with the piezomagnetic one, we find that the latter is far more dominant (SASAI, 1985). We only have to take into account the piezomagnetic effect in the case of the "cold" tectonic events.

The analytic solution for a point source was obtained by the systematic use of the Fourier transforms. This method was also applied to dislocation problems in an elastic half-space (SASAI, 1980). Actually, magnetic changes caused by strike-slip faulting of a vertical rectangular fault were solved analytically. The point source solution has a fundamental use in the construction of Green's functions for dislocation problems.

However, SASAI's (1979) solution is too crude an approximation to an actual magma chamber. Since the magma chamber has a finite size, a substantial volume of host rocks around the chamber should be thermally demagnetized. In connection with this issue, a discrepancy has been noticed between DAVIS' (1976) and SASAI's (1979) results. This might be due to the difference in the source size between the two. The cause of this discrepancy has, however, so far been unknown.

SUZUKI and OSHIMAN (1990) followed DAVIS' (1976) work and reproduced his results numerically. They were also able to reproduce SASAI's (1979) results when the chamber size was reduced in size to a point source. Spatial patterns of total intensity changes, for example, are very different between those of DAVIS (1986) and SASAI (1979). However, SUZUKI and OSHIMAN (1990) pointed out that the numerical and analytic results are almost coincident if the Curie depth is shallower than that of the source. This implies that the effect of the source and its size is crucial in the piezomagnetic field of the Mogi model. It is therefore important to investigate the transitional behavior of the solution by varying the radius of a non-magnetic sphere, including the pressure source.

Numerical volume integrals require of much CPU time. Moreover, it is difficult to estimate computational errors in the case of 3-D integrations. We present here an analytical solution for a finite spherical source. Investigating the characteristics of the solution, we found an unexpected result: the limiting case of reducing the source sphere radius to zero does not agree with SASAI's (1979) solution. This arises from differences in the evaluation of improper integrals due to a divergent stress component.

A different approach to that of SASAI (1979) was practiced in this paper to obtain a point source solution. We called it the type II solution, while the original solution by SASAI (1979) was called the type I. We will then derive an analytic expression for the finite spherical source, i.e. the type III solution. It can no longer be expressed with elementary functions. The type III solution was reduced to a one-dimensional integral containing complete elliptic integrals. We can numerically integrate it with a sufficient accuracy. Computation time is remarkably cut down as compared with the 3-D volume integrals.

We find that this solution reproduces DAVIS' (1976), and SUZUKI and OsHIMAN's (1990) results. The behavior of the solution was fully investigated by changing the radius 
of the source sphere as well as the depth of the Curie point isotherm. The limiting case of the type III solution where the source radius reduces to zero, coincides with the type II solution. The cause of the discrepancy between the type I and II solutions results from the neglection of some portion of piezomagnetic constituents produced in the magnetized region.

\section{Formulation of the Problem}

Let us take the Cartesian coordinates as shown in Fig. 1. A semi-infinite elastic medium occupies $z>0$. It is also assumed that a layer from $z=0$ to $z=H$ is uniformly magnetized. We suppose a small sphere with radius $a$, centered at a point $A(0,0, D)$, has a hydrostatic pressure $\Delta P$ applied from inside. Since the spherical pressure source is regarded as a magma chamber, a concentric spherical shell with radius $b$ is assumed to be thermally demagnetized and non-magnetic. Its boundary may coincide with the source sphere: i.e. $b \geq a$. The Curie point isotherm may lie either above, below or even across the demagnetized sphere. The point source problem indicates the case in which $a$ and $b$ are infinitesimally small.

The displacement field of the Mogi model is equivalent to that produced by a strain nucleus called the center of dilatation (MINDLIN and CHENG, 1950; YAMAKAWA, 1955). It is given as follows:

$$
\begin{aligned}
& u_{x}=\frac{C}{2 \mu}\left\{\frac{x}{R_{1}^{3}}+\frac{\lambda+3 \mu}{\lambda+\mu} \frac{x}{R_{2}^{3}}-\frac{6 x z(z+D)}{R_{2}^{5}}\right\}, \\
& u_{y}=\frac{C}{2 \mu}\left\{\frac{y}{R_{1}^{3}}+\frac{\lambda+3 \mu}{\lambda+\mu} \frac{y}{R_{2}^{3}}-\frac{6 y z(z+D)}{R_{2}^{5}}\right\}, \\
& u_{z}=\frac{C}{2 \mu}\left\{\frac{z-D}{R_{1}^{3}}+\frac{(\lambda-\mu) z-(\lambda+3 \mu) D}{(\lambda+\mu) R_{2}^{3}}-\frac{6 z(z+D)^{2}}{R_{2}^{5}}\right\},
\end{aligned}
$$

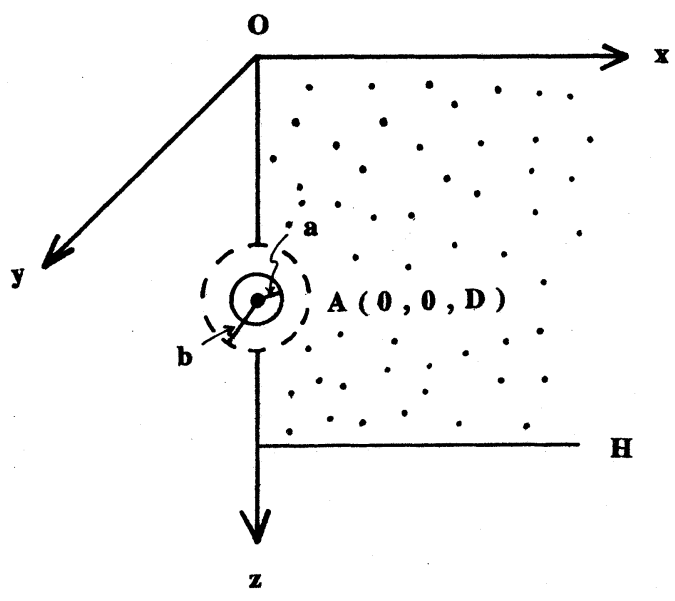

Fig. 1. Coordinate system, a hydrostatically pumped pressure source at $A(0,0, D)$ with radius $a$, the thermally demagnetized zone with radius $b$ and the Curie point isotherm at a depth $H$. 
where

$$
\begin{aligned}
& R_{1}=\sqrt{x^{2}+y^{2}+(z-D)^{2}}, \\
& R_{2}=\sqrt{x^{2}+y^{2}+(z+D)^{2}},
\end{aligned}
$$

$\lambda$ and $\mu$ are Lamè's constants, while $C$ is the moment of the strain nucleus. Under the condition that $a$ is sufficiently small compared to $D$, the moment $C$ is given by

$$
C=-\frac{1}{2} a^{3} \Delta P
$$

We follow the standard stress convention: compression is negative. This is opposite to the definition by SASAI (1979). The first terms on the right-hand side of Eqs. (2.1) correspond to the displacement field due to a center of dilatation in an infinite medium, while the remainders represent the effects of the free surface. We will hereafter specify the former $\boldsymbol{u}^{(\mathrm{A})}$ and the latter $\boldsymbol{u}^{(\mathrm{B})}$. In the point source problem displacements become singular at point $A(0,0, D)$.

Differentiation of Eqs. (2.1) gives the stress field. Applying the piezomagnetic law for 3-D stress state (SASAI, 1980), we can obtain components of the stress-induced magnetization at an arbitrary point. They are given, in general, as follows:

$$
\Delta \boldsymbol{J}=\beta \boldsymbol{S} \cdot \boldsymbol{J}
$$

where

$$
\begin{aligned}
& S_{j k}=\frac{3}{2} \tau_{j k}-\frac{1}{2} \delta_{j k} \Theta=\mu\left\{\frac{3}{2}\left(\frac{\partial u_{j}}{\partial x_{k}}+\frac{\partial u_{k}}{\partial x_{j}}\right)-\delta_{j k} \Delta\right\}, \\
& \Theta=\tau_{x x}+\tau_{y y}+\tau_{z z}, \quad \Delta=\operatorname{div} \boldsymbol{u}
\end{aligned}
$$

in which $\beta$ is the stress sensitivity and $\tau_{i j}$ each of the components of the stress tensor.

The piezomagnetic components associated with the Mogi model are given explicitly in the following:

$$
\begin{aligned}
S_{x x}=\frac{C}{2}\left\{3\left(\frac{1}{R_{1}^{3}}-\frac{3 x^{2}}{R_{1}^{5}}\right)+3 \frac{\lambda+3 \mu}{\lambda+\mu}\left(\frac{1}{R_{2}^{3}}-\frac{3 x^{2}}{R_{2}^{5}}\right)\right. & \\
& \left.+\frac{4 \mu}{\lambda+\mu}\left(\frac{1}{R_{2}^{3}}-\frac{3(z+D)^{2}}{R_{2}^{5}}\right)-18 z(z+D)\left(\frac{1}{R_{2}^{5}}-\frac{5 x^{2}}{R_{2}^{7}}\right)\right\}, \\
S_{x y}= & \frac{C}{2}\left\{-\frac{9 x y}{R_{1}^{5}}-9 \frac{\lambda+3 \mu}{\lambda+\mu} \frac{x y}{R_{2}^{5}}+\frac{90 x y z(z+D)}{R_{2}^{7}}\right\}, \\
S_{x z}= & \frac{C}{2}\left\{-\frac{9 x(z-D)}{R_{1}^{5}}-\frac{9 x(3 z+D)}{R_{2}^{5}}+\frac{90 x z(z+D)^{2}}{R_{2}^{7}}\right\}, \\
S_{y x}= & S_{x y}, \quad S_{y y}=S_{x x}(x \leftrightarrow y), \quad S_{y z}=S_{z y},
\end{aligned}
$$




$$
\begin{aligned}
S_{z x}=S_{x z} & \\
S_{z y}=\frac{C}{2} & \left\{-\frac{9 y(z-D)}{R_{1}^{5}}-\frac{9 y(3 z+D)}{R_{2}^{5}}+\frac{90 y z(z+D)^{2}}{R_{2}^{7}}\right\}, \\
S_{z z}=\frac{C}{2} & \left\{3\left(\frac{1}{R_{1}^{3}}-\frac{3(z-D)^{2}}{R_{1}^{5}}\right)+\frac{3 \lambda+\mu}{\lambda+\mu} \frac{1}{R_{2}^{3}}\right. \\
& \left.-3\left(\frac{21 \lambda+19 \mu}{\lambda+\mu} z+\frac{3 \lambda+\mu}{\lambda+\mu} D\right) \frac{z+D}{R_{2}^{5}}+\frac{90 z(z+D)^{3}}{R_{2}^{7}}\right\} .
\end{aligned}
$$

The first terms on the right-hand side of Eqs. (2.7) represent the magnetization produced by $\boldsymbol{u}^{(\mathrm{A})}$, and the remainders the magnetization produced by $\boldsymbol{u}^{(\mathrm{B})}$. Obviously, the first terms diverge as we approach point A.

SASAI (1979) derived an expression for the piezomagnetic law to be used in the case of the axially symmetric stress field. Piezomagnetic components according to that formula, i.e. Eq. (32) in SASAI (1979), look slightly different from Eqs. (2.7), the former containing $x^{2} / r^{2}$ etc. However, we can easily find that both are identical after some manipulations. force as;

The potential of the magnetic field produced by $\Delta J(\boldsymbol{r})$ is given by the dipole law of

$$
W\left(\boldsymbol{r}_{0}\right)=\iiint_{V} \Delta J(\boldsymbol{r}) \cdot \nabla\left(\frac{1}{\rho}\right) \mathrm{d} \boldsymbol{r},
$$

where

$$
\rho=\left|\boldsymbol{r}-\boldsymbol{r}_{0}\right|
$$

and $\boldsymbol{r}_{0}$ is the position vector at which the magnetic field is measured. $V$ indicates the whole magnetized area. In the case of the point source problem, the right-hand side of Eq. (2.8) becomes an improper integral owing to the first terms in Eqs. (2.7).

Both DAVIS' (1976) and SASAI's (1979) calculations are based on the formula (2.8). Instead; we will use a modified formula, i.e. a surface integral representation for the piezomagnetic potential (SASAI, 1983), which is given by

$$
\begin{aligned}
W_{k}\left(\boldsymbol{r}_{0}\right)=C_{k} \iint_{S}\left[\left\{-\frac{\partial u_{k}(\boldsymbol{r})}{\partial n}\right.\right. & \left.+\frac{2(\lambda+\mu)}{3 \lambda+2 \mu} \Delta \boldsymbol{m}^{(k)} \cdot \boldsymbol{n}\right\} \frac{1}{\rho} \\
& \left.+\left\{\boldsymbol{u}_{k}(\boldsymbol{r})\right\} \frac{\partial}{\partial n}\left(\frac{1}{\rho}\right)\right] \mathrm{d} S
\end{aligned}
$$

where

$$
\Delta m_{l}^{(k)}=\frac{3}{2}\left(\frac{\partial u_{k}}{\partial x_{l}}+\frac{\partial u_{l}}{\partial x_{k}}\right)-\delta_{k l} \operatorname{div} \boldsymbol{u}
$$




$$
C_{k}=\frac{1}{2} \beta J_{k} \mu \frac{3 \lambda+2 \mu}{\lambda+\mu}
$$

$J_{k}$ is the $k$-th component of the uniform magnetization. $S$ indicates the whole surface surrounding the magnetized volume $V$, and $\boldsymbol{n}$ outward normal to $S$. This formula was deduced from Eq. (2.8) with the aid of Green's theorem.

\section{Point Source Problem}

Let us consider the point source problem. We subdivide the magnetized crust into two parts: a layer from the earth's surface to the source depth $(0<z<D)$ and a layer from the source depth to the Curie point isotherm $(D<z<H)$. See Fig. 2(a). Integrating Eq. (2.8) for respective regions and adding them, we obtain SASAI's (1979) solution. To apply the formula (2.10), we may take account of the next four plane surfaces; i.e. the earth's surface $(z=0)$, a horizontal plane just above the source point $(z=D-\varepsilon)$, a plane just below the source $(z=D+\varepsilon)$, and the Curie point isotherm $(z=H)$. Actually, SASAI (1983) recalculated the piezomagnetic potential of the Mogi model with the aid of Eq. (2.10) and arrived at the same result as the one obtained through volumetric integrals. The contribution from the point source is defined as a limit diminishing the distance between two surfaces, $z=D-\varepsilon$ and $z=D+\varepsilon$. We called it the type I solution.

We may consider another way of integration which is usually adopted for improper integrals. It is, to integrate Eq. (2.8) over a region, except for a small sphere with radius $\varepsilon$ centered at $(0,0, D)$, and then to approach $a=b=\varepsilon$ to zero. See Fig. 2(b). We called it the type II solution. However, it is much easier to achieve surface integrations according to Eq. (2.10) rather than the volumetric ones in Eq. (2.8). We are to evaluate contributions from the earth's surface, the Curie point isotherm and a spherical surface with radius $\varepsilon$. The former two have already appeared in the type I solution. Let us investigate these solutions systematically.

\subsection{Type I solution}

SASAI (1983) obtained this solution through surface integrations. Only the results are quoted here for the sake of comparison. The piezomagnetic potential produced by the $k$-th component of the uniform magnetization consists of three parts:

(a) TYPE I

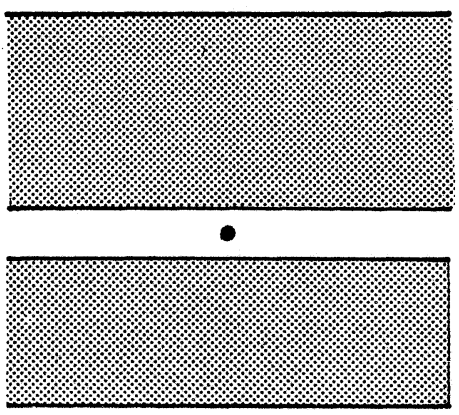

(b) TYPE II

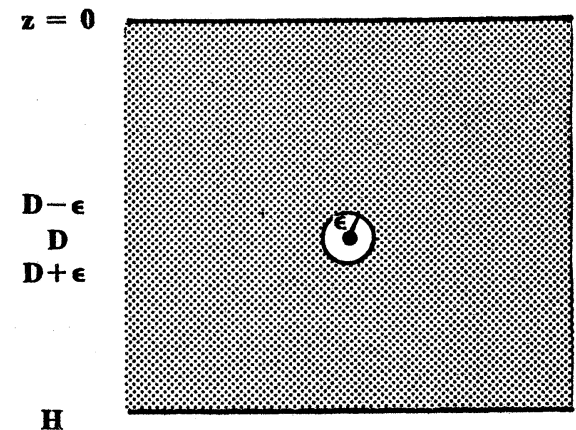

Fig. 2. A schematic representation of integration area (shaded) for (a) type I and (b) type II solution. 


$$
W_{k}^{(\mathrm{I})}=W_{k}^{(0)}+W_{k}^{(\mathrm{P})}+W_{k}^{(\mathrm{H})}
$$

in which the superscripts indicate contributions from the free surface $(0)$, the pressure source $(\mathrm{P})$, and the Curie point isotherm $(\mathrm{H})$ respectively. Because of the symmetrical nature of the Mogi model, we may take the $x$ axis in the magnetic north direction without a loss of generality. $W_{k}^{(0)}$ and $W_{k}^{(\mathrm{H})}$ are convolution integrals, which can be evaluated by the Fourier transform method. We obtain:

$$
\begin{aligned}
& W_{x}^{(0)}=0, \\
& W_{z}^{(0)}=0,
\end{aligned}
$$

and

$$
\begin{aligned}
& \frac{2 \mu}{C} W_{x}^{(\mathrm{H})}=4 \pi C_{x}\left[-\frac{\mu}{3 \lambda+2 \mu} \frac{x_{0}}{\rho_{3}^{3}}+\frac{6(\lambda+\mu)}{3 \lambda+2 \mu} H \frac{3 x_{0} D_{3}}{\rho_{3}^{5}}\right. \\
& +\left\{\begin{aligned}
\left.-\frac{3(\lambda+\mu)}{3 \lambda+2 \mu} \frac{x_{0}}{\rho_{2}^{3}}\right] & (H>D) \\
\left.\frac{\mu}{3 \lambda+2 \mu} \frac{x_{0}}{\rho_{1}^{3}}\right] & (H<D),
\end{aligned}\right. \\
& \frac{2 \mu}{C} W_{z}^{(\mathrm{H})}=4 \pi C_{z}\left[\frac{\mu}{3 \lambda+2 \mu} \frac{D_{3}}{\rho_{3}^{3}}+\frac{6(\lambda+\mu)}{3 \lambda+2 \mu} H\left(-\frac{1}{\rho_{3}^{3}}+\frac{3 D_{3}^{2}}{\rho_{3}^{5}}\right)\right. \\
& + \begin{cases}\left.-\frac{3(\lambda+\mu)}{3 \lambda+2 \mu} \frac{D_{2}}{\rho_{2}^{3}}\right] & (H>D) \\
\left.-\frac{\mu}{3 \lambda+2 \mu} \frac{D_{1}}{\rho_{1}^{3}}\right] & (H<D),\end{cases}
\end{aligned}
$$

where

$$
\begin{aligned}
& \rho_{i}=\left(x_{0}^{2}+y_{0}^{2}+D_{i}^{2}\right)^{1 / 2}, \\
& D_{1}=D-z_{0}, \quad D_{2}=2 H-D-z_{0}, \quad D_{3}=2 H+D-z_{0} .
\end{aligned}
$$

Since outward normals of the surface $z=D-\varepsilon$ and $z=D+\varepsilon$ face each other, $W_{k}^{(\mathrm{P})}$ is given by;

$$
W_{k}^{(\mathrm{P})}=\lim _{\varepsilon \rightarrow 0}\left\{W_{k}^{(H=D-\varepsilon)}-W_{k}^{(H=D+\varepsilon)}\right\} .
$$

Substituting Eqs. (3.3), we obtain:

$$
\frac{2 \mu}{C} W_{x}^{(\mathrm{P})}=4 \pi C_{x} \frac{3 \lambda+4 \mu}{3 \lambda+2 \mu} \frac{x_{0}}{\rho_{1}^{3}},
$$




$$
\frac{2 \mu}{C} W_{z}^{(\mathrm{P})}=4 \pi C_{z} \frac{D_{1}}{\rho_{1}^{3}}
$$

Those terms containing $\rho_{3}$ in Eqs. (3.3) are contributions from $\boldsymbol{u}^{(\mathrm{B})}$. They cancel with each other in the limiting operation (3.5). However, all the terms arising from $\boldsymbol{u}^{(\mathrm{A})}$ do not always survive in Eqs. (3.6). A close inspection shows that the single layer potential remains in $W_{x}^{(\mathrm{P})}$ and that the double layer does in $W_{z}^{(\mathrm{P})}$. Thus, the magnitude of the source sphere contribution is different for the horizontal and vertical magnetization case respectively.

Combining Eqs. (3.2), (3.3) and (3.6), we obtain the type I solution as follows:

$$
\begin{gathered}
\frac{2 \mu}{C} W_{x}^{(\mathrm{I})}=4 \pi C_{x}\left[\frac{\mu}{3 \lambda+2 \mu}\left(\frac{x_{0}}{\rho_{1}^{3}}-\frac{x_{0}}{\rho_{3}^{3}}\right)+\frac{6(\lambda+\mu)}{3 \lambda+2 \mu} H \frac{3 x_{0} D_{3}}{\rho_{3}^{5}}\right. \\
+\left\{\begin{array}{cc}
\left.\frac{3(\lambda+\mu)}{3 \lambda+2 \mu}\left(\frac{x_{0}}{\rho_{1}^{3}}-\frac{x_{0}}{\rho_{2}^{3}}\right)\right] & (H>D) \\
0 & (H<D),
\end{array}\right. \\
\frac{2 \mu}{C} W_{z}^{(\mathrm{I})}=4 \pi C_{z}\left[-\frac{\mu}{3 \lambda+2 \mu}\left(\frac{D_{1}}{\rho_{1}^{3}}-\frac{D_{3}}{\rho_{3}^{3}}\right)+\frac{6(\lambda+\mu)}{3 \lambda+2 \mu} H\left(-\frac{1}{\rho_{3}^{3}}+\frac{3 D_{3}^{2}}{\rho_{3}^{5}}\right)\right. \\
+\left\{\begin{array}{cc}
\left.\frac{3(\lambda+\mu)}{3 \lambda+2 \mu}\left(\frac{D_{1}}{\rho_{1}^{3}}-\frac{D_{2}}{\rho_{2}^{3}}\right)\right] & (H>D) \\
0 \quad & (H<D) .
\end{array}\right.
\end{gathered}
$$

\subsection{Type II solution}

We evaluate the surface integral (2.10) over a spherical surface of radius $b$ as shown in Fig. 3. Let us take the spherical coordinates $\left(R_{1}, \theta, \phi\right)$ with its origin at $A(0,0, D)$ and its polar axis in the $z$ direction. An observation point $Q\left(r_{0}\right)$ in the free space is given by $Q\left(\rho_{1}, \theta_{0}, \phi_{0}\right)$, while a moving point $P(\boldsymbol{r})=P(b, \theta, \phi)$ sweeps the source sphere. Then we have

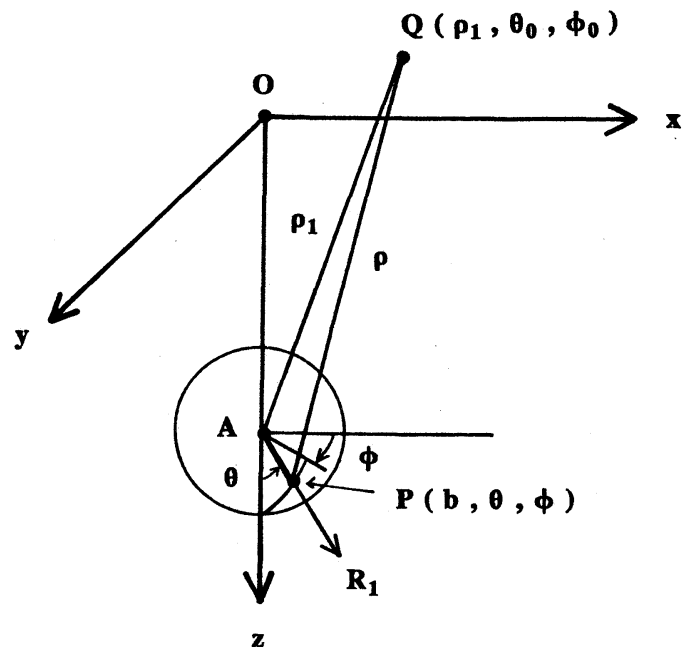

Fig. 3. Spherical coordinate system for integration over a spherical cap. 


$$
\rho=\sqrt{\rho_{1}^{2}+R_{1}^{2}-2 \rho_{1} R_{1} \cos \gamma}
$$

in which $\gamma$ is the angle between AP and AQ. $\cos \gamma$ is given by

$$
\cos \gamma=\cos \theta \cos \theta_{0}+\sin \theta \sin \theta_{0} \cos \left(\phi-\phi_{0}\right)
$$

$\rho_{1}$ and $R_{1}$ are defined by Eq. (3.4a) and Eq. (2.2a) in the original Cartesian coordinates. We write the contribution from the spherical surface $R_{1}=b$ as $W_{k}^{(\mathrm{K})}$. Since the outward normal is given by $\boldsymbol{n}=-\boldsymbol{e}_{R_{1}}$, we have

$$
W_{k}^{(\mathrm{K})}=C_{k} \int_{0}^{2 \pi} \mathrm{d} \phi \int_{0}^{\pi}\left[F_{1 k} \cdot G_{1}+F_{2 k} \cdot G_{2}\right] \sin \theta \mathrm{d} \theta,
$$

where

$$
\begin{aligned}
& F_{1 k}=b^{2}\left[\frac{\partial u_{k}}{\partial R_{1}}-\frac{3(\lambda+\mu)}{3 \lambda+2 \mu} \Delta m^{(k)} \cdot \boldsymbol{e}_{R_{1}}\right]_{R_{1}=b}, \\
& F_{2 k}=b^{2}\left[u_{k}\right]_{R_{1}=b}
\end{aligned}
$$

and

$$
\begin{aligned}
G_{1} & =\left[\frac{1}{\rho}\right]_{R_{1}=b}, \\
G_{2} & =\left[-\frac{\partial}{\partial R_{1}}\left(\frac{1}{\rho}\right)\right]_{R_{1}=b} .
\end{aligned}
$$

$F_{1 k}$ 's and $F_{2 k}$ 's give density distributions of single and double layer potentials respectively. We may discriminate those arising from $\boldsymbol{u}^{(\mathrm{A})}$ and $\boldsymbol{u}^{(\mathrm{B})}$.

$$
\begin{aligned}
\frac{2 \mu}{C} F_{1 x}^{(\mathrm{A})}=\frac{2(3 \lambda+4 \mu)}{3 \lambda+2 \mu} \frac{\sin \theta \cos \phi}{b} & \\
\frac{2 \mu}{C} F_{1 x}^{(\mathrm{B})}=2 b^{2} \sin \theta \cos \phi[ & \left(\frac{2(3 \lambda+7 \mu)}{3 \lambda+2 \mu}-\frac{\lambda+3 \mu}{\lambda+\mu}\right) \frac{1}{S^{3}} \\
& +3\left\{2\left(s^{2}+\frac{3 \lambda+4 \mu}{\lambda+\mu} D s+\frac{\lambda+2 \mu}{\lambda+\mu} D^{2}\right)\right. \\
& \left.-2 \frac{(9 \lambda+10 \mu)}{3 \lambda+2 \mu}(s+D)^{2}+\frac{12(\lambda+\mu)}{3 \lambda+2 \mu} D^{2}\right\} \frac{1}{S^{5}} \\
& \left.+\frac{30(3 \lambda+4 \mu)}{3 \lambda+2 \mu} \frac{D s(s+D)^{2}}{S^{7}}\right] \\
\frac{2 \mu}{C} F_{2 x}^{(\mathrm{A})}=\sin \theta \cos \phi, &
\end{aligned}
$$




$$
\begin{gathered}
\frac{2 \mu}{C} F_{2 x}^{(\mathrm{B})}=b^{3} \sin \theta \cos \phi\left\{\frac{\lambda+3 \mu}{\lambda+\mu} \frac{1}{S^{3}}-\frac{6 s(s+D)}{S^{5}}\right\} \\
\frac{2 \mu}{C} F_{1 z}^{(\mathrm{A})}=\frac{2(3 \lambda+4 \mu)}{3 \lambda+2 \mu} \frac{\cos \theta}{b}, \\
\frac{2 \mu}{C} F_{1 z}^{(\mathrm{B})}=2 b\left[\left(-\frac{\lambda-\mu}{\lambda+\mu} s+\frac{\lambda+5 \mu}{\lambda+\mu} D\right) \frac{1}{S^{3}}+\left(10 s+3 \frac{2 \lambda+5 \mu}{3 \lambda+2 \mu} D\right) \frac{1}{S^{3}}\right. \\
+3\left(\frac{\lambda-\mu}{\lambda+\mu} s-\frac{\lambda+3 \mu}{\lambda+\mu} D\right) \frac{D(s+D)}{S^{5}} \\
\quad+3\left(2 s^{2}+7 D s+D^{2}\right) \frac{s+D}{S^{5}}-\frac{9(\lambda+\mu)}{3 \lambda+2 \mu} \frac{(13 s+D)(s+D)^{2}}{S^{5}} \\
\quad-3\left(\frac{21 \lambda+11 \mu}{3 \lambda+2 \mu} s+\frac{3 \lambda-7 \mu}{3 \lambda+2 \mu} D\right) \frac{s^{2}-D^{2}}{S^{5}} \\
\left.\quad-\frac{30 D s(s+D)^{3}}{S^{7}}+\frac{180(\lambda+\mu)}{3 \lambda+2 \mu} \frac{s^{2}(s+D)^{3}}{S^{7}}\right] \\
\frac{2 \mu}{C} F_{2 z}^{(\mathrm{A})}=\cos \theta, \\
\frac{2 \mu}{C} F_{2 z}^{(\mathrm{B})}=b^{2}\left[\left(\frac{\lambda-\mu}{\lambda+\mu} s-\frac{\lambda+3 \mu}{\lambda+\mu} D\right)-\frac{6 s(s+D)^{2}}{S^{5}}\right]
\end{gathered}
$$

where $s$ and $S$ are nothing but $z$ and $R_{2}$ in the polar coordinates, when P moves on the source sphere:

$$
\begin{aligned}
& s=b \cos \theta+D, \\
& S=\sqrt{b^{2}+4 b D \cos \theta+4 D^{2}}=\sqrt{b^{2}+4 D s} .
\end{aligned}
$$

$\rho^{-1}$ can be expanded as follows:

$$
\frac{1}{\rho}=\sum_{n=0}^{\infty} \frac{R_{1}^{n}}{\rho_{1}^{n+1}} P_{n}(\cos \gamma) .
$$

Thus the single layer potential $G_{1}$ on the sphere with unit intensity and the double layer one $G_{2}$ can be given by;

$$
\begin{aligned}
G_{1} & =\sum_{n=0}^{\infty} \frac{b^{n}}{\rho_{1}^{n+1}} P_{n}(\cos \gamma), \\
G_{2} & =\sum_{n=1}^{\infty} \frac{n b^{n-1}}{\rho_{1}^{n+1}} P_{n}(\cos \gamma) .
\end{aligned}
$$

To compute (3.10), we may use the following integration theorem of spherical harmonics. 


$$
\int_{0}^{2 \pi} \mathrm{d} \phi \int_{0}^{\pi} Y_{n}(\theta, \phi) P_{m}(\cos \gamma) \sin \theta \mathrm{d} \theta=\left\{\begin{array}{cc}
\frac{4 \pi}{2 n+1} Y_{n}\left(\theta_{0}, \phi_{0}\right) & (n=m) \\
0 & (n \neq m) .
\end{array}\right.
$$

$Y_{n}(\theta, \phi)$ is an arbitrary spherical harmonic function.

By applying this theorem, we can readily evaluate integrals involving the $\boldsymbol{u}^{(\mathrm{A})}$ term. As for the (B) terms we have an infinite series of $P_{n}\left(\cos \theta_{0}\right)$. This solution is less useful because of its poor convergency. However, all the (B) terms are multiplied by a factor $b$, so that they vanish in the limit of $b$ approaching zero. As far as the point source problem is concerned, we may disregard the contribution from $\boldsymbol{u}^{(\mathrm{B})}$. We will give different expressions for the (B) terms in the next section. follows:

With the aid of the theorem (3.17) we obtain the pressure source contribution as

$$
\begin{aligned}
\frac{2 \mu}{C} W_{x}^{(\mathrm{K})} & =C_{x}\left\{\frac{2(3 \lambda+4 \mu)}{3 \lambda+2 \mu} \frac{1}{b} \sin \theta_{0} \cos \phi_{0} \frac{b}{\rho_{1}^{2}}-\sin \theta_{0} \cos \phi_{0} \frac{1}{\rho_{1}^{2}}\right\} \times \frac{4 \pi}{3} \\
& =4 \pi C_{x} \frac{\lambda+2 \mu}{3 \lambda+2 \mu} \frac{\sin \theta_{0} \cos \phi_{0}}{\rho_{1}^{2}}=4 \pi C_{x} \frac{\lambda+2 \mu}{3 \lambda+2 \mu} \frac{x_{0}}{\rho_{1}^{3}}
\end{aligned}
$$

and quite similarly

$$
\begin{aligned}
\frac{2 \mu}{C} W_{z}^{(\mathrm{K})} & =C_{z}\left\{\frac{2(3 \lambda+4 \mu)}{3 \lambda+2 \mu} \frac{1}{b} \cos \theta_{0} \frac{b}{\rho_{1}^{2}}-\cos \theta_{0} \frac{1}{\rho_{1}^{2}}\right\} \times \frac{4 \pi}{3} \\
& =4 \pi C_{z} \frac{\lambda+2 \mu}{3 \lambda+2 \mu} \frac{\cos \theta_{0}}{\rho_{1}^{3}}=4 \pi C_{z} \frac{\lambda+2 \mu}{3 \lambda+2 \mu} \frac{-D_{1}}{\rho_{1}^{3}} .
\end{aligned}
$$

The solutions (3.18) have nothing to do with the source radius $b$. Thus they survive when the radius $b$ approaches zero.

Combining Eqs. (3.2), (3.3) and (3.18), we obtain the type II solution as follows:

$$
\begin{gathered}
\frac{2 \mu}{C} W_{x}^{(\mathrm{II})}=4 \pi C_{x}\left[\frac{\mu}{3 \lambda+2 \mu}\left(\frac{x_{0}}{\rho_{1}^{3}}-\frac{x_{0}}{\rho_{3}^{3}}\right)+\frac{6(\lambda+\mu)}{3 \lambda+2 \mu} H \frac{3 x_{0} D_{3}}{\rho_{3}^{5}}\right. \\
+\left\{\begin{array}{ccc}
\left.\frac{\lambda+\mu}{3 \lambda+2 \mu}\left(\frac{x_{0}}{\rho_{1}^{3}}-\frac{3 x_{0}}{\rho_{2}^{3}}\right)\right] & (H>D) \\
0 & (H<D),
\end{array}\right. \\
\frac{2 \mu}{C} W_{z}^{(\mathrm{II})}=4 \pi C_{z}\left[-\frac{\mu}{3 \lambda+2 \mu}\left(\frac{D_{1}}{\rho_{1}^{3}}-\frac{D_{3}}{\rho_{3}^{3}}\right)+\frac{6(\lambda+\mu)}{3 \lambda+2 \mu} H\left(-\frac{1}{\rho_{3}^{3}}+\frac{3 D_{3}^{2}}{\rho_{3}^{5}}\right)\right. \\
+\left\{\begin{array}{ccc}
\left.-\frac{\lambda+\mu}{3 \lambda+2 \mu}\left(\frac{D_{1}}{\rho_{1}^{3}}+\frac{3 D_{2}}{\rho_{2}^{3}}\right)\right] & (H>D) \\
0 & (H<D) .
\end{array}\right.
\end{gathered}
$$


As shown in Eqs. (3.18), both of the source terms produced by $J_{x}$ and $J_{z}$ are identical. This feature is naturally expected because $\boldsymbol{u}^{(\mathrm{A})}$ has a spherical symmetry. In other words, the type I solution, Eqs. (3.6), lacks of something. We should investigate the cause of the discrepancy between the two. Moreover, in the type II solution, the source term $W_{k}^{(\mathrm{K})}$ suddenly emerges when the Curie depth $H$ increases to cross over the source depth $D$. We have to search into such a transitional behavior for the type II solution. These problems will be discussed in Section 6.

The moment of the pressure source $C$ is related to the maximum uplift just above the source $\Delta h_{\max }$ as follows:

$$
C=\frac{\lambda+\mu}{\lambda+2 \mu} \mu D^{2} \Delta h_{\max }
$$

where

$$
\Delta h_{\max }=\frac{\lambda+2 \mu}{2(\lambda+\mu)} \frac{-\Delta P}{\mu}\left(\frac{a}{D}\right)^{3} D .
$$

Equation (3.20) is favorable for the point source solution, because we do not need values for $\Delta P$ and $a$ explicitly. For a given model of finite spherical source with parameters $a$, $\Delta P$, and $D$, we may calculate $C$ for a corresponding point source solution with the aid of Eq. (3.20) and Eq. (3.21). DAVIS (1976) computed piezomagnetic changes for the Kilauea Volcano with the model parameters listed in Table 1 . In this case we have $\Delta h_{\max }=0.47 \mathrm{~m}$. Total field changes associated with the corresponding point source model are shown in Fig. 4(a) for the type I, and in Fig. 4(b) for the type II solution respectively. Both are apparently quite different. Which type of solution is appropriate?

\section{Finite Spherical Source Problem}

Now let us consider the case when the Curie point isotherm intersects the thermally demagnetized source sphere. We cannot apply the theorem (3.17) to evaluate $W_{k}^{(\mathrm{K})}$, i.e. integrals over a spherical cap. Nor can we use Fourier transforms to calculate $W_{k}^{(\mathrm{H})}$, because the horizontal plane with infinite extent has a circular hole. We are now to formulate such a case. The formulation allows for integration either over the whole spherical surface or an infinite plane without a hole, as a special case.

Table 1. Model parameters for piezomagnetic computations of the Mogi model after DAvis (1976).

\begin{tabular}{lll}
\hline Depth of the center of the source sphere & $D$ & $4 \mathrm{~km}$ \\
Radius of the spherical pressure source & $a$ & $1 \mathrm{~km}$ \\
(= Radius of the demagnetized sphere) & $(=b)$ & $1 \mathrm{~km}$ \\
Internal hydrostatic pressure & $\Delta P$ & $1 \mathrm{kbar}$ \\
Stress sensitivity & $\beta$ & $2.0 \times 10^{-4} \mathrm{bar}^{-1}$ \\
Total magnetization & $J$ & $5 \mathrm{~A} / \mathrm{m}$ \\
Geomagnetic inclination (Kilauea Volcano) & $I_{0}$ & $35^{\circ}$ \\
Geomagnetic declination (Kilauea Volcano) & $D_{0}$ & $11^{\circ}$ east \\
\hline
\end{tabular}




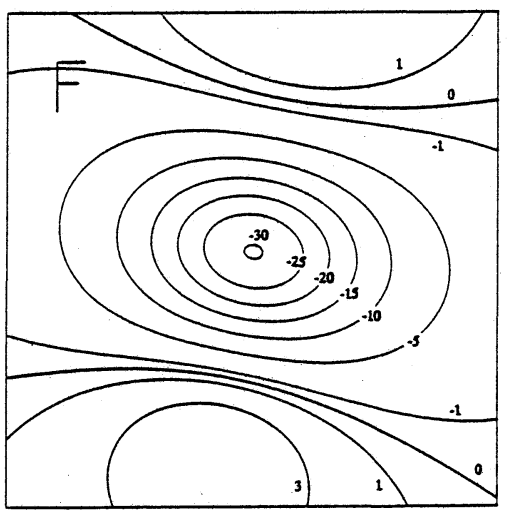

(a)

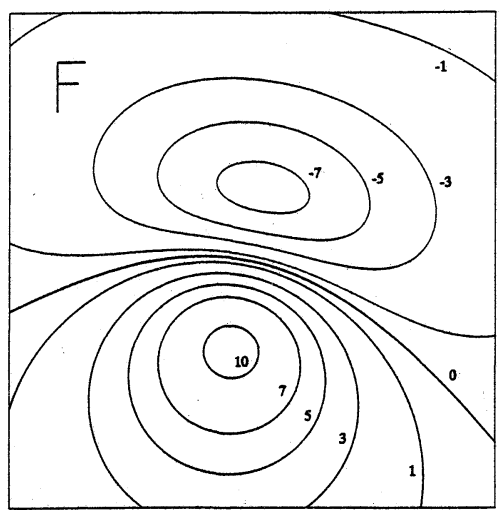

(b)

Fig. 4. Computed total intensity changes for Kilauea Volcano with the same model parameters as DAVIS' (1976) for (a) type I solution and (b) type II solution. Unit in nT.

\subsection{Contribution from spherical cap}

We take the same spherical coordinates as those in Fig. 3. The integral over a spherical cap is defined in Eq. (3.10) by exchanging the lower limit of $\theta$ from 0 to $\Theta$ as below;

$$
\Theta=\left\{\begin{array}{cc}
\cos ^{-1} \frac{H-D}{b} & (|H-D|<b) \\
0 & (H-D \geq b) .
\end{array}\right.
$$

Performing integrations with respect to $\phi$, we obtain the following expression in the place of Eq. (3.10):

$$
W_{k}^{(\mathrm{K})}=C_{k} \int_{\theta}^{\pi} \sin \theta \mathrm{d} \theta\left[F_{1 k}(\theta) \cdot G_{1 k}\left(\theta ; \boldsymbol{r}_{0}\right)+F_{2 k}(\theta) \cdot G_{2 k}\left(\theta ; \boldsymbol{r}_{0}\right)\right]
$$

where

$$
\begin{aligned}
& F_{1 x}(\theta)=\left\{F_{1 x}^{(\mathrm{A})}+F_{1 x}^{(\mathrm{B})}\right\} / \cos \phi, \\
& F_{2 x}(\theta)=\left\{F_{2 x}^{(\mathrm{A})}+F_{2 x}^{(\mathrm{B})}\right\} / \cos \phi, \\
& F_{1 z}(\theta)=\left\{F_{1 z}^{(\mathrm{A})}+F_{1 z}^{(\mathrm{B})}\right\}, \\
& F_{2 z}(\theta)=\left\{F_{2 z}^{(\mathrm{A})}+F_{2 z}^{(\mathrm{B})}\right\},
\end{aligned}
$$

and

$$
G_{1 x}\left(\theta ; \boldsymbol{r}_{0}\right)=\int_{0}^{2 \pi} \frac{\cos \phi}{\rho_{b}} \mathrm{~d} \phi=2 \cos \phi_{0} \cdot \Phi_{2},
$$




$$
\begin{aligned}
G_{2 x}\left(\theta ; \boldsymbol{r}_{0}\right) & =\int_{0}^{2 \pi} \frac{b-\rho_{1} \cos \gamma}{\rho_{b}^{3}} \cos \phi \mathrm{d} \phi \\
& =2 \cos \phi_{0}\left\{2\left(b-\zeta_{0} \cos \theta\right) \cdot \Phi_{4}-r_{0} \sin \theta \cdot\left(\Phi_{3}+\Phi_{5}\right)\right\} \\
G_{1 z}\left(\theta ; \boldsymbol{r}_{0}\right) & =\int_{0}^{2 \pi} \frac{1}{\rho_{b}} \mathrm{~d} \phi=2 \Phi_{1} \\
G_{2 z}\left(\theta ; \boldsymbol{r}_{0}\right) & =\int_{0}^{2 \pi} \frac{b-\rho_{1} \cos \gamma}{\rho_{b}^{3}} \mathrm{~d} \phi \\
& =2\left(b-\zeta_{0} \cos \theta\right) \cdot \Phi_{3}-2 r_{0} \sin \theta \cdot \Phi_{4} .
\end{aligned}
$$

$\rho_{b}$ is given as $\rho\left(R_{1}=b\right)$ in Eq. (3.8). $F_{1 k}^{(\mathrm{A})}$ 's and $F_{2 k}^{(\mathrm{B})}$ 's have already been given in Eqs. (3.13). Notice that a common factor $\cos \phi$ is removed from $F_{1 X}$ and $F_{2 X}$ to be included within $G_{1 X}$ and $G_{2 X}$. $\Phi_{n}$ 's in Eqs. (4.4) are defined by the following integrals:

$$
\begin{array}{ll}
\Phi_{1}=\int_{0}^{\pi} \frac{1}{\rho_{\psi}} \mathrm{d} \psi, & \Phi_{2}=\int_{0}^{\pi} \frac{\cos \psi}{\rho_{\psi}} \mathrm{d} \psi, \\
\Phi_{3}=\int_{0}^{\pi} \frac{1}{\rho_{\psi}^{3}} \mathrm{~d} \psi, & \Phi_{4}=\int_{0}^{\pi} \frac{\cos \psi}{\rho_{\psi}^{3}} \mathrm{~d} \psi, \quad \Phi_{5}=\int_{0}^{\pi} \frac{\cos 2 \psi}{\rho_{\psi}^{3}} \mathrm{~d} \psi .
\end{array}
$$

$\rho_{\psi}$ is rewritten from $\rho_{b}$ as a function of $\psi=\phi-\phi_{0}$ :

$$
\begin{aligned}
\rho_{\psi} & =\left(b^{2}-2 b \rho_{1} \cos \theta \cos \theta_{0}+\rho_{1}^{2}-2 b \rho_{1} \sin \theta \sin \theta_{0} \cos \psi\right)^{1 / 2} \\
& =\left\{b^{2} \sin ^{2} \theta-2 r_{0} b \sin \theta \cos \psi+r_{0}^{2}+\left(b \cos \theta-\zeta_{0}\right)^{2}\right\}^{1 / 2}
\end{aligned}
$$

where

$$
\zeta_{0}=\rho_{1} \cos \theta_{0}=z_{0}-D
$$

$r_{0}$ is horizontal distance of the observation point in the original Cartesian coordinates: i.e. $r_{0}=\sqrt{x_{0}^{2}+y_{0}^{2}}$.

Since $\Phi_{n}$ 's cannot be expressed with elementary functions, we have to make a numerical integration with respect to $\theta$. We seek to obtain expressions for the magnetic field components in the form of integrals with respect to $\theta$, by differentiating potentials (4.2). Thus we have

$$
\begin{aligned}
& X_{x}^{(\mathrm{K})}=C_{x} \int_{\Theta}^{\pi} \sin \theta \mathrm{d} \theta\left[F_{1 x}(\theta) \cdot G_{1 x}^{X}+F_{2 x}(\theta) \cdot G_{2 x}^{X}\right], \\
& Y_{x}^{(\mathrm{K})}=C_{x} \int_{\Theta}^{\pi} \sin \theta \mathrm{d} \theta\left[F_{1 x}(\theta) \cdot G_{1 x}^{Y}+F_{2 x}(\theta) \cdot G_{2 x}^{Y}\right], \\
& Z_{x}^{(\mathrm{K})}=C_{x} \int_{\Theta}^{\pi} \sin \theta \mathrm{d} \theta\left[F_{1 x}(\theta) \cdot G_{1 x}^{Z}+F_{2 x}(\theta) \cdot G_{2 x}^{Z}\right], \\
& X_{z}^{(\mathrm{K})}=C_{z} \int_{\Theta}^{\pi} \sin \theta \mathrm{d} \theta\left[F_{1 z}(\theta) \cdot G_{2 z}^{X}+F_{2 z}(\theta) \cdot G_{2 z}^{X}\right],
\end{aligned}
$$




$$
\begin{aligned}
& Y_{z}^{(\mathrm{K})}=C_{z} \int_{\Theta}^{\pi} \sin \theta \mathrm{d} \theta\left[F_{1 z}(\theta) \cdot G_{1 z}^{Y}+F_{2 z}(\theta) \cdot G_{2 z}^{Y}\right], \\
& Z_{z}^{(\mathrm{K})}=C_{z} \int_{\Theta}^{\pi} \sin \theta \mathrm{d} \theta\left[F_{1 z}(\theta) \cdot G_{1 z}^{Z}+F_{2 x}(\theta) \cdot G_{2 z}^{Z}\right],
\end{aligned}
$$

$G_{1 x}^{l}$ s $(l=X, Y, Z)$ are given as follows:

$$
\begin{aligned}
G_{1 x}^{X} & =2 r_{0} \cos ^{2} \phi_{0} \cdot \Phi_{4}-b \sin \theta \cdot\left(\Phi_{3}+\cos 2 \phi_{0} \cdot \Phi_{5}\right), \\
G_{1 x}^{Y} & =\sin 2 \phi_{0}\left(r_{0} \cdot \Phi_{4}-b \sin \theta \cdot \Phi_{5}\right) \\
G_{1 x}^{Z} & =2 \cos \phi_{0}\left(\zeta_{0}-b \cos \theta\right) \cdot \Phi_{4} .
\end{aligned}
$$

$G_{2 x}^{l}$ 's $(l=X, Y, Z)$ are given as follows:

$$
\begin{aligned}
& G_{2 x}^{X}= \sin \theta \cdot\left(\Phi_{3}+\cos 2 \phi_{0} \cdot \Phi_{5}\right)+6 r_{0} \cos ^{2} \phi_{0}\left(b-\zeta_{0} \cos \theta\right) \cdot \Phi_{7} \\
&-3 r_{0}^{2} \cos ^{2} \phi_{0} \sin \theta \cdot\left(\Phi_{6}+\Phi_{8}\right)-3 b \sin \phi\left(b-\zeta_{0} \cos \theta\right) \cdot\left(\Phi_{6}+\cos 2 \phi_{0} \cdot \Phi_{8}\right) \\
&+3 b r_{0} \sin ^{2} \theta\left\{\left(\frac{1}{2}+\cos ^{2} \phi_{0}\right) \cdot \Phi_{7}+\frac{1}{2} \cos 2 \phi_{0} \cdot \Phi_{9}\right\} \\
& G_{2 x}^{Y}= \sin 2 \phi_{0}\left\{\sin \theta \cdot \Phi_{5}+3 r_{0}\left(b-\zeta_{0} \cos \theta\right) \cdot \Phi_{7}-\frac{3}{2} r_{0}^{2} \sin \theta \cdot\left(\Phi_{6}+\Phi_{8}\right)\right. \\
&\left.-3 b \sin \theta\left(b-\zeta_{0} \cos \theta\right) \cdot \Phi_{8}+\frac{3}{2} b r_{0} \sin ^{2} \theta \cdot\left(\Phi_{7}+\Phi_{9}\right)\right\} \\
& G_{2 x}^{Z}= \cos \phi_{0}\left\{2 \cos \theta \cdot \Phi_{4}+6\left(b-\zeta_{0} \cos \theta\right)\left(\zeta_{0}-b \cos \theta\right) \cdot \Phi_{7}\right. \\
&\left.-3 r_{0} \sin \theta\left(\zeta_{0}-b \cos \theta\right) \cdot\left(\Phi_{6}+\Phi_{8}\right)\right\} .
\end{aligned}
$$

$G_{1 z}^{l}$ 's $(l=X, Y, Z)$ are given as follows:

$$
\begin{aligned}
G_{1 z}^{X} & =2 \cos \phi_{0}\left(r_{0} \cdot \Phi_{3}-b \sin \theta \cdot \Phi_{4}\right), \\
G_{1 z}^{Y} & =2 \sin \phi_{0}\left(r_{0} \cdot \Phi_{3}-b \sin \theta \cdot \Phi_{4}\right), \\
G_{1 z}^{Z} & =2\left(\zeta_{0}-b \cos \theta\right) \cdot \Phi_{3} .
\end{aligned}
$$

$G_{2 z}^{l}$ 's $(l=X, Y, Z)$ are given as follows:

$$
\begin{aligned}
G_{2 z}^{X}=2 \cos \phi_{0}\{ & \sin \theta \cdot \Phi_{4}+3 r_{0}\left(b-\zeta_{0} \cos \theta\right) \cdot \Phi_{6} \\
& -3 \sin \theta\left(r_{0}^{2}+b\left(b-\zeta_{0} \cos \theta\right)\right) \cdot \Phi_{7} \\
& \left.+\frac{3}{2} b r_{0} \sin ^{2} \theta \cdot\left(\Phi_{6}+\Phi_{8}\right)\right\}
\end{aligned}
$$




$$
\begin{aligned}
& G_{2 z}^{Y}=2 \sin \phi_{0}\{ \sin \theta \cdot \Phi_{4}+3 r_{0}\left(b-\zeta_{0} \cos \theta\right) \cdot \Phi_{6} \\
&-3 \sin \theta\left(r_{0}^{2}+b\left(b-\zeta_{0} \cos \theta\right)\right) \cdot \Phi_{7} \\
&\left.+\frac{3}{2} b r_{0} \sin ^{2} \theta \cdot\left(\Phi_{6}+\Phi_{8}\right)\right\} \\
& G_{2 z}^{Z}=2 \cos \theta \cdot \Phi_{3}+6\left(b-\zeta_{0} \cos \theta\right)\left(\zeta_{0}-b \cos \theta\right) \cdot \Phi_{6} \\
&-6 r_{0} \sin \theta\left(\zeta_{0}-b \cos \theta\right) \cdot \Phi_{7} .
\end{aligned}
$$

In addition to Eqs. (4.5) and (4.6), we need to follow the $\Phi_{n}$ 's.

$$
\begin{array}{ll}
\Phi_{6}=\int_{0}^{\pi} \frac{1}{\rho_{\psi}^{5}} \mathrm{~d} \psi, & \Phi_{7}=\int_{0}^{\pi} \frac{\cos \psi}{\rho_{\psi}^{5}} \mathrm{~d} \psi, \\
\Phi_{8}=\int_{0}^{\pi} \frac{\cos 2 \psi}{\rho_{\psi}^{5}} \mathrm{~d} \psi, & \Phi_{9}=\int_{0}^{\pi} \frac{\cos 3 \psi}{\rho_{\psi}^{5}} \mathrm{~d} \psi .
\end{array}
$$

$\Phi_{n}$ 's can be expressed with complete elliptic integrals. Following EASON et al. (1955), we treat these integrals in a unified way. We first reduce $\Phi_{n}$ to an integral of LipschitzHankel type, defined as

$$
I(m, n ; l)=\int_{0}^{\infty} J_{m}(a t) J_{n}(b t) \mathrm{e}^{-c t} t^{l} \mathrm{~d} t
$$

$J_{m}$ and $J_{n}$ are Bessel functions. We should replace $a, b$, and $c$ with the following variables.

$$
a=b \sin \theta, \quad b=r_{0}, \quad c=b \cos \theta+D-z_{0} .
$$

Transformation of $\Phi_{n}$ into the Lipschitz-Hankel integral is achieved in Appendix A. Next, we will express those with complete elliptic integrals. Some integrals of this type in Eq. (4.14) were not given explicitly by EASON et al. (1955). All the integrals necessary for the present purpose are tabulated in Appendix B. They are eventually represented by combination of complete elliptic integrals of the first and second kind. Numerical approximations formulas are available for these two functions (HASTINGS, 1955).

Thus integrands in Eqs. (4.9) can be calculated accurately as well as rapidly. As for one-dimensional integral of analytic functions, we have a useful algorithm for the numerical integration, i.e. the double exponential formula (TAKAHASI and MORI, 1974). Now we have an analytic expression for integrals over a spherical cap and an effective way of integrating it: the problem is solved.

\subsection{Contribution from the Curie surface with a circular hole}

We take the cylindrical coordinates $(u, \phi, z)$, of which origin is $(0,0, H)$, an intersection point of the $z$ axis with the Curie depth surface. See Fig. 5. Let us consider the surface integral over the magnetized portion of the Curie surface. The integral in radial direction should be carried out from $u_{0}$ to infinity. $u_{0}$ is given by 


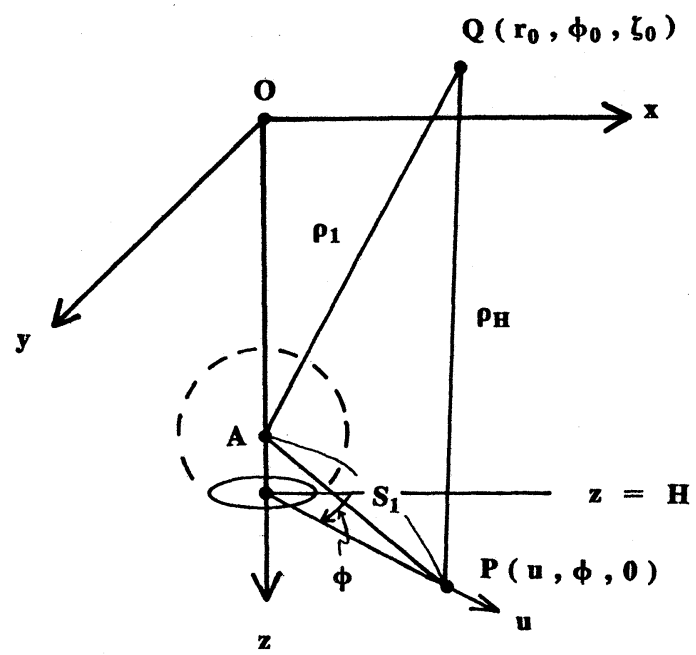

Fig. 5. Cylindrical coordinate system for integration over the Curie surface with a circular hole.

$$
u_{0}=\left\{\begin{array}{cc}
\sqrt{b^{2}-(H-D)^{2}} & (|H-D|<b) \\
0 & (|H-D| \geq b)
\end{array}\right.
$$

We first conduct integrations with respect to $\phi$ and obtain

$$
\begin{aligned}
W_{k}^{(\mathrm{H})}=C_{k} \int_{u_{0}}^{\infty}[ & \left\{f_{1 k}^{(\mathrm{A})}(u)+f_{1 k}^{(\mathrm{B})}(u)\right\} \cdot g_{1 k}\left(u ; \boldsymbol{r}_{0}\right) \\
& \left.+\left\{f_{2 k}^{(\mathrm{A})}(u)+f_{2 k}^{(\mathrm{B})}(u)\right\} \cdot g_{2 k}\left(u ; \boldsymbol{r}_{0}\right)\right] u \mathrm{~d} u
\end{aligned}
$$

where

$$
\begin{aligned}
& \frac{2 \mu}{C} f_{1 x}^{(\mathrm{A})}=3 u\left\{-\frac{3 \lambda+4 \mu}{3 \lambda+2 \mu} \frac{H-D}{S_{1}^{5}}\right\}, \\
& \frac{2 \mu}{C} f_{1 x}^{(\mathrm{B})}=3 u\left[\left\{-\left(\frac{\lambda-\mu}{\lambda+\mu}+\frac{6 \mu}{3 \lambda+2 \mu}\right) H+\left(\frac{\lambda+3 \mu}{\lambda+\mu}-\frac{2 \mu}{3 \lambda+2 \mu}\right) D\right\} \frac{1}{S_{2}^{5}}\right. \\
& \left.+\frac{10(3 \lambda+4 \mu)}{3 \lambda+2 \mu} \frac{H(H+D)^{2}}{S_{2}^{7}}\right] \text {, } \\
& \frac{2 \mu}{C} f_{2 x}^{(\mathrm{A})}=\frac{u}{S_{1}^{3}} \\
& \frac{2 \mu}{C} f_{2 x}^{(\mathrm{B})}=u\left[\frac{\lambda+3 \mu}{\lambda+\mu} \frac{1}{S_{2}^{3}}-\frac{6 H(H+D)}{S_{2}^{5}}\right] \text {, } \\
& \frac{2 \mu}{C} f_{1 z}^{(\mathrm{A})}=\frac{3 \lambda+4 \mu}{3 \lambda+2 \mu}\left\{\frac{1}{S_{1}^{3}}-\frac{3(H-D)^{2}}{S_{1}^{5}}\right\} \text {, }
\end{aligned}
$$




$$
\begin{aligned}
\frac{2 \mu}{C} f_{1 z}^{(\mathrm{B})}= & \frac{3 \lambda+4 \mu}{3 \lambda+2 \mu}\left[\frac{\lambda-\mu}{\lambda+\mu} \frac{1}{S_{2}^{3}}-3\left(\frac{7 \lambda+5 \mu}{\lambda+\mu} H+\frac{\lambda-\mu}{\lambda+\mu} D\right) \frac{H+D}{S_{2}^{5}}\right. \\
& \left.+\frac{30 H(H+D)^{3}}{S_{2}^{7}}\right] \\
& +\frac{8 \mu}{3 \lambda+2 \mu}\left[\frac{1}{S_{2}^{3}}-\frac{3(H+D)^{2}}{S_{2}^{5}}\right], \\
\frac{2 \mu}{C} f_{2 z}^{(\mathrm{A})}= & \frac{H-D}{S_{1}^{3}}, \\
\frac{2 \mu}{C} f_{2 z}^{(\mathrm{B})}= & \left(\frac{\lambda-\mu}{\lambda+\mu} H-\frac{\lambda+3 \mu}{\lambda+\mu} D\right) \frac{1}{S_{2}^{3}}-\frac{6 H(H+D)^{2}}{S_{2}^{5}}
\end{aligned}
$$

in which

$$
S_{1}=\sqrt{u^{2}+(H-D)^{2}}, \quad S_{2}=\sqrt{u^{2}+(H+D)^{2}}
$$

The single layer potential $g_{1 k}$ 's and the double layer potential $g_{2 k}$ 's are given by

$$
\begin{aligned}
& g_{1 x}=\int_{0}^{2 \pi} \frac{\cos \phi}{\rho_{H}} \mathrm{~d} \phi=2 \cos \phi_{0} \cdot \Phi_{2}, \\
& g_{2 x}=\int_{0}^{2 \pi} \frac{\left(z_{0}-H\right) \cos \phi}{\rho_{H}^{3}} \mathrm{~d} \phi=2\left(z_{0}-H\right) \cos \phi_{0} \cdot \Phi_{4}, \\
& g_{1 z}=\int_{0}^{2 \pi} \frac{1}{\rho_{H}} \mathrm{~d} \phi=2 \Phi_{1}, \\
& g_{2 z}=\int_{0}^{2 \pi} \frac{\left(z_{0}-H\right)}{\rho_{H}^{3}} \mathrm{~d} \phi=2\left(z_{0}-H\right) \cdot \Phi_{3},
\end{aligned}
$$

where

$$
\begin{aligned}
\rho_{H} & =\left\{u^{2}+\left(H-z_{0}\right)^{2}+x_{0}^{2}+y_{0}^{2}-2 u\left(x_{0} \cos \phi+y_{0} \sin \phi\right)\right\}^{1 / 2} \\
& =\left\{u^{2}-2 r_{0} u \cos \psi+r_{0}^{2}+\left(H-z_{0}\right)^{2}\right\}^{1 / 2}
\end{aligned}
$$

Comparing Eq. (4.22) with Eq. (4.7), we find that the integrals in Eqs. (4.21) are formally quite identical with those in Eqs. (4.4). In this case we only have to replace $a, b$, and $c$ with the following variables:

$$
a=u, \quad b=r_{0}, \quad c=H-z_{0}
$$

Differentiation of potentials gives us the magnetic field components as:

$$
X_{x}^{(\mathrm{H})}=C_{x} \int_{u_{0}}^{\infty}\left[f_{1 x}(u) \cdot g_{1 x}^{X}+f_{2 x}(u) \cdot g_{2 x}^{X}\right] u \mathrm{~d} u,
$$




$$
\begin{aligned}
& Y_{x}^{(\mathrm{H})}=C_{x} \int_{u_{0}}^{\infty}\left[f_{1 x}(u) \cdot g_{1 x}^{Y}+f_{2 x}(u) \cdot g_{2 x}^{Y}\right] u \mathrm{~d} u, \\
& Z_{x}^{(\mathrm{H})}=C_{x} \int_{u_{0}}^{\infty}\left[f_{1 x}(u) \cdot g_{1 x}^{Z}+f_{2 x}(u) \cdot g_{2 x}^{Z}\right] u \mathrm{~d} u, \\
& X_{z}^{(\mathrm{H})}=C_{z} \int_{u_{0}}^{\infty}\left[f_{1 z}(u) \cdot g_{1 z}^{X}+f_{2 z}(u) \cdot g_{2 z}^{X}\right] u \mathrm{~d} u, \\
& Y_{z}^{(\mathrm{H})}=C_{z} \int_{u_{0}}^{\infty}\left[f_{1 z}(u) \cdot g_{1 z}^{Y}+f_{2 z}(u) \cdot g_{2 z}^{Y}\right] u \mathrm{~d} u, \\
& Z_{z}^{(\mathrm{H})}=C_{z} \int_{u_{0}}^{\infty}\left[f_{1 z}(u) \cdot g_{1 z}^{Z}+f_{2 z}(u) \cdot g_{2 z}^{Z}\right] u \mathrm{~d} u .
\end{aligned}
$$

$g_{1 x}^{l}$ 's $(l=X, Y, Z)$ are given by

$$
\begin{aligned}
& g_{1 x}^{X}=2 r_{0} \cos ^{2} \phi_{0} \cdot \Phi_{4}-u \cdot\left(\Phi_{3}+\cos 2 \phi_{0} \cdot \Phi_{5}\right), \\
& g_{1 x}^{Y}=\sin 2 \phi_{0} \cdot\left(r_{0} \cdot \Phi_{4}-u \cdot \Phi_{5}\right), \\
& g_{1 x}^{Z}=2\left(z_{0}-H\right) \cos \phi_{0} \cdot \Phi_{4} .
\end{aligned}
$$

$g_{2 x}^{l}$ 's $(l=X, Y, Z)$ are given by

$$
\begin{aligned}
& g_{2 x}^{X}=3\left(z_{0}-H\right)\left[2 r_{0} \cos ^{2} \phi_{0} \cdot \Phi_{7}-u \cdot\left(\Phi_{6}+\cos 2 \phi_{0} \cdot \Phi_{8}\right)\right] \\
& g_{2 x}^{Y}=3\left(z_{0}-H\right) \sin 2 \phi_{0} \cdot\left(r_{0} \cdot \Phi_{7}-u \cdot \Phi_{8}\right) \\
& g_{2 x}^{Z}=2 \cos \phi_{0} \cdot\left(-\Phi_{4}+3\left(z_{0}-H\right)^{2} \cdot \Phi_{7}\right)
\end{aligned}
$$

$g_{1 z}^{l}$ 's $(l=X, Y, Z)$ are given by

$$
\begin{aligned}
& g_{1 z}^{X}=2 \cos \phi_{0} \cdot\left(r_{0} \cdot \Phi_{3}-u \cdot \Phi_{4}\right), \\
& g_{1 z}^{Y}=2 \sin \phi_{0} \cdot\left(r_{0} \cdot \Phi_{3}-u \cdot \Phi_{4}\right), \\
& \mathrm{g}_{1 z}^{Z}=2\left(z_{0}-H\right) \cdot \Phi_{3} .
\end{aligned}
$$

$g_{2 z}^{l}$ 's $(l=X, Y, Z)$ are given by

$$
\begin{aligned}
& g_{2 z}^{X}=6\left(z_{0}-H\right) \cos \phi_{0} \cdot\left(r_{0} \cdot \Phi_{6}-u \cdot \Phi_{7}\right) \\
& g_{1 z}^{Y}=6\left(z_{0}-H\right) \sin \phi_{0} \cdot\left(r_{0} \cdot \Phi_{6}-u \cdot \Phi_{7}\right), \\
& g_{2 z}^{Z}=-2 \Phi_{3}+6\left(z_{0}-H\right)^{2} \cdot \Phi_{6} .
\end{aligned}
$$

We can numerically calculate integrals in Eqs. (4.24) with the aid of the DEF. Thus we have managed to evaluate the contribution of an infinite plane boundary with a circular hole.

If the Curie surface does not intersect the demagnetized sphere, we should always evaluate the integrals over a plane without a hole, i.e. Eq. (4.18) in which $u_{0}=0$. We have already obtained analytic solutions for this particular integrals with the aid of the Fourier transform method. The integral (4.18) must be reduced to Eqs. (3.3). Actually this is true, 
which is proved in the Appendix C. For $|H-D|>b$, we may omit elaborate numerical integrations in Eqs. (4.24).

We may numerically calculate the magnetic field by applying Eqs. (4.24) and compare with those obtained by differentiating Eqs. (3.3). Thus we can examine the accuracy of numerical computations. Comparing the numerical results through Eqs. (4.24) with those through (3.3), we find that both are coincident within the first 5 digits. The double exponential formula guarantees an accuracy of up to the double precision, provided integrands are given with double precision and the interval of integration is appropriately subdivided. Hence errors in our computation are mainly caused by insufficient accuracy of the approximation formulas for complete elliptic integrals (i.e. single precision). However, even the accuracy to 5 digits is quite sufficient for the purpose.

When the source sphere is entirely buried within the magnetized region, the contribution from $\boldsymbol{u}^{(\mathrm{A})}$ in the spherical surface integral (4.2) is much simplified. We find that $\Phi_{n}$ 's are reduced to elementary functions along a symmetry axis. If we choose a line connecting the dilation center $A(0,0, D)$ and the observation point $Q\left(x_{0}, y_{0}, z_{0}\right)$ as a new polar axis, we see that the axially symmetric nature of $\boldsymbol{u}^{(\mathrm{A})}$ still holds. The integration is easily achieved: those arising from $\boldsymbol{u}^{(\mathrm{A})}$ are reduced to Eqs. (3.18). The proof is trivial. However, we should conduct numerical integrations for the $\boldsymbol{u}^{(\mathrm{B})}$ constituents even in the case of the entire spherical surface integral.

\section{Characteristics of the Finite Source Solution}

In this section we will investigate some characteristics of the finite source solution. We present how the surface magnetic field varies by increasing the depth of the Curie point isotherm. The behavior of the solution by diminishing the source radius is also shown.

\subsection{Curie depth dependency of the magnetic field at the origin}

DAVIS (1976) and SASAI (1979) reported how the magnetic field at the origin $(0,0,0)$ just above the center of dilatation varies by increasing Curie depth $H$. At this particular point, only two field components, i.e. $X_{x}$ and $Z_{z}$, are non-zero. SUZUKI and OSHIMAN (1990) reexamined Davis' numerical calculations by subdividing the medium with a sufficiently small size of elementary volume. Their model parameters are very similar to those of DAVIS (1976) in Table 1, with the only exception of the depth $D$ and the radius $b$ of the source sphere, i.e. $D=12 \mathrm{~km}$ and $b=3 \mathrm{~km}$ respectively. We follow here their model parameters for the sake of comparison.

Figure 6 shows the Curie depth dependency of $X_{x}$ and $Z_{z}$ at the origin. This figure agrees very well with SUZUKI and OSHIMAN's (1990) results (Fig. 7(b) of their paper). This implies that their numerical calculation is very accurate. Davis' result is now verified again through an analytic approach. As DAVIS (1976) has already pointed out, the piezomagnetic changes become to their maximum when the Curie depth $H$ is around the source depth $D$. They decrease to certain values with the increasing depth of $H$. This characteristic is quite different from the type I solution, in which the maximum (absolute) changes are attained at $H$ infinity (SASAI, 1979; see Fig. 14(a)). Figure 6 shows that the contribution of the vertical magnetization $J_{z}$ is much more dominant than that of the horizontal $J_{x}$. This feature was also noticed by DAVIS (1976). 


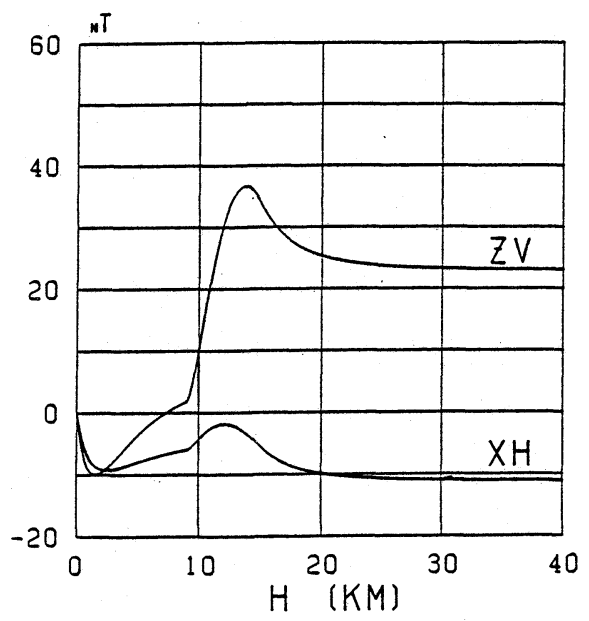

Fig. 6. Two non-zero magnetic components at the origin with increasing depth of $H$. Model parameters are the same as SUZUKI and OshIMAN's (1990). XH and ZV indicate $X_{x}$ and $Z_{z}$ component respectively.

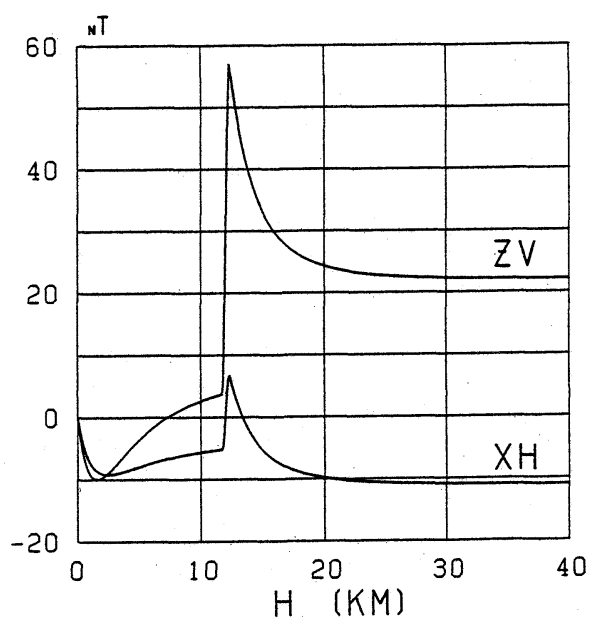

(a)

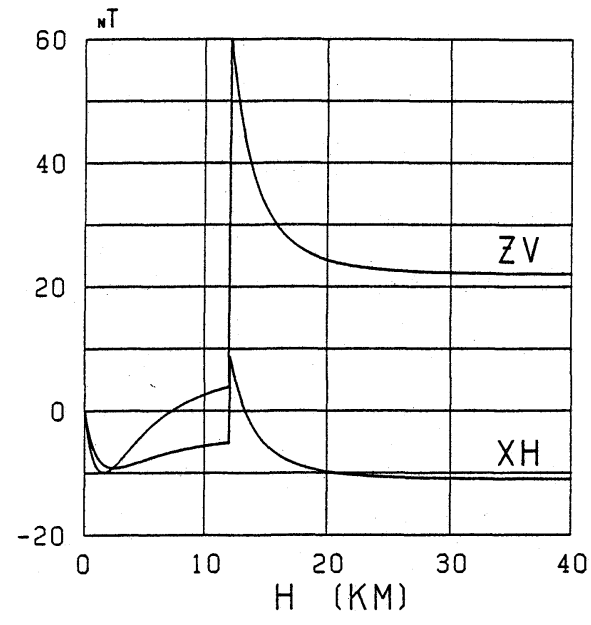

(b)

Fig. 7. Curie depth dependency curves of the magnetic components at the origin for (a) $b=300 \mathrm{~m}$ and (b) $b=30 \mathrm{~m}$, respectively.

$\Phi_{n}$ 's involved in the solution can be reduced to irrational functions when the observation point is located along the symmetry axis; i.e. $r_{0}=0$. Hence we can conduct integrations in Eqs. (4.8a), (4.8f), (4.23a), and (4.23f) analytically to obtain solutions expressed with elementary functions. They were actually used to examine the validity of numerical computations through the DEF. Since analytic expressions for the magnetic field at the origin are rather complicated, they are not presented here.

Let us investigate the influence of the source size. Figure 7(a) shows the curve of the Curie depth dependency for $b=300 \mathrm{~m}$, while Fig. 7(b) is that for $b=30 \mathrm{~m}$. The other 

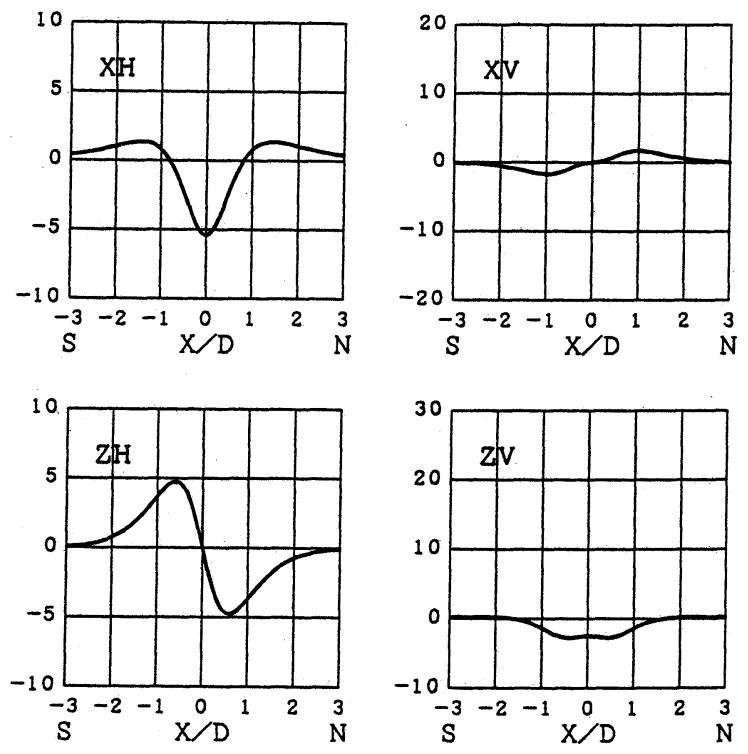

(a)
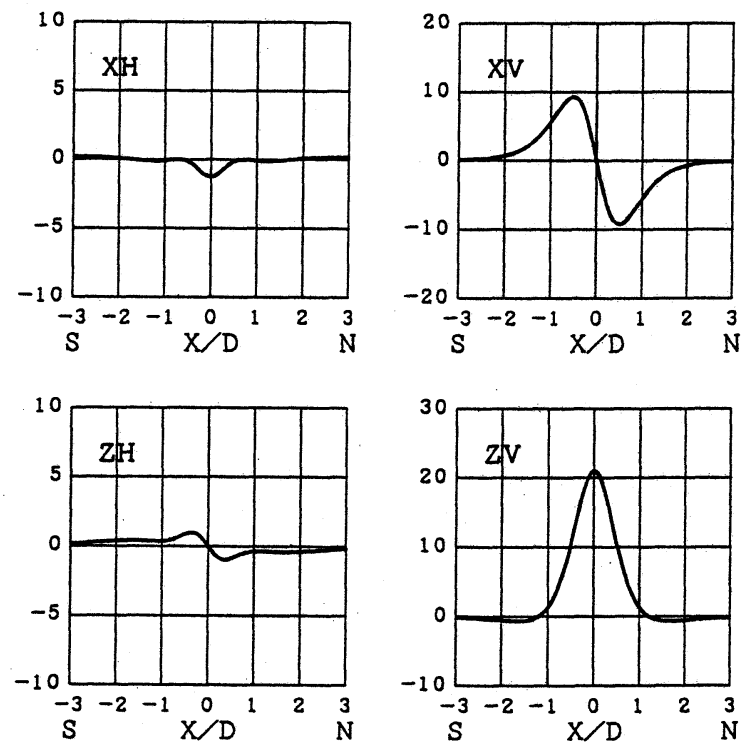

(b)

Fig. 8. Four non-zero magnetic components along the N-S meridian for (a) $H=5 \mathrm{~km}$, (b) $H=12 \mathrm{~km}$ and (c) $H=20 \mathrm{~km}$. Unit in the ordinate is $\mathrm{nT}$. 

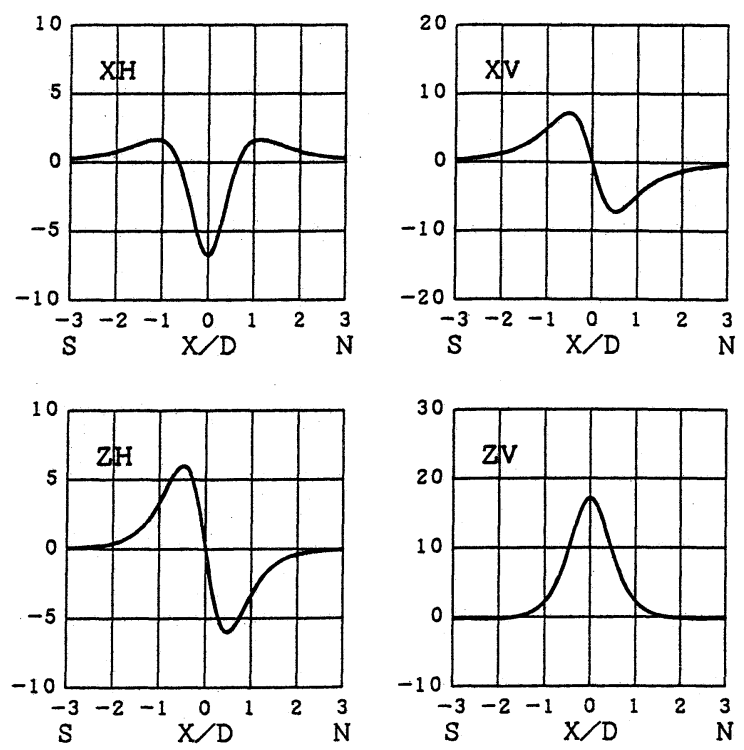

(c)

Fig. 8. (continued).

model parameters are the same as those in Fig. 6. The special feature of the curve in Fig. 6 is preserved, somewhat exaggerated as $b$ decreases. The peak values of the surface magnetic field around $H=D$ kept constant for smaller values of $b$. The $H$ dependency curve was never observed to drastically convert into that of the type I solution (i.e. Fig. 14(a)).

\subsection{Magnetic field along the $\mathrm{N}-\mathrm{S}$ meridian}

Figure 8 shows the magnetic field components along the North-South meridian line. Model parameters are the same as SUZUKI and OSHIMAN's (1990), particularly for (a) $H=5 \mathrm{~km}$, (b) $H=12 \mathrm{~km}$ and (c) $H=20 \mathrm{~km}$. There are four non-zero components, i.e. $X_{H}$, $Z_{H}, X_{V}$ and $Z_{V}$. They depend on the inclination of the ambient field. Hence $X_{H}$ and $Z_{H}$ are given in units of $(C / 2 \mu) C_{x}$, while $X_{V}$ and $Z_{V}$ in units of $(C / 2 \mu) C_{z}$. The horizontal extent is $\pm 3 D(= \pm 36 \mathrm{~km})$ across the origin. As we have already seen in Fig. 6 , the $Z_{V}$ component steeply grows up as $H$ passes the source sphere. This component is dominant for $H \geq D$.

Let us examine how the magnetic field varies in the case of $H=D(=12 \mathrm{~km})$ as the source radius $b$ diminishes. Figures 9(a), (b), and (c) show the field components along the $\mathrm{N}-\mathrm{S}$ meridian for $b=1 \mathrm{~km}, 300 \mathrm{~m}$ and $30 \mathrm{~m}$ respectively. Comparing those with Fig. 8(b), we find that the polarity reverses between $b=3 \mathrm{~km}$ and $1 \mathrm{~km}$ in the $X_{H}$ and $Z_{H}$ components. Actually, the transition occurs around $b=1.6 \mathrm{~km}$. On the other hand, $X_{V}$ and $Z_{V}$ components indicate a typical feature of those for a vertical downward magnetic dipole. The magnetic field produced by $J_{x}$ is much weaker than that of $J_{z}$. In the midlatitudes, the influence of $J_{z}$ is greatly dominant: the complicate variation of $X_{H}$ and $Z_{H}$ along with the radius $b$ does not so much affect the overall pattern of the composite magnetic field. 

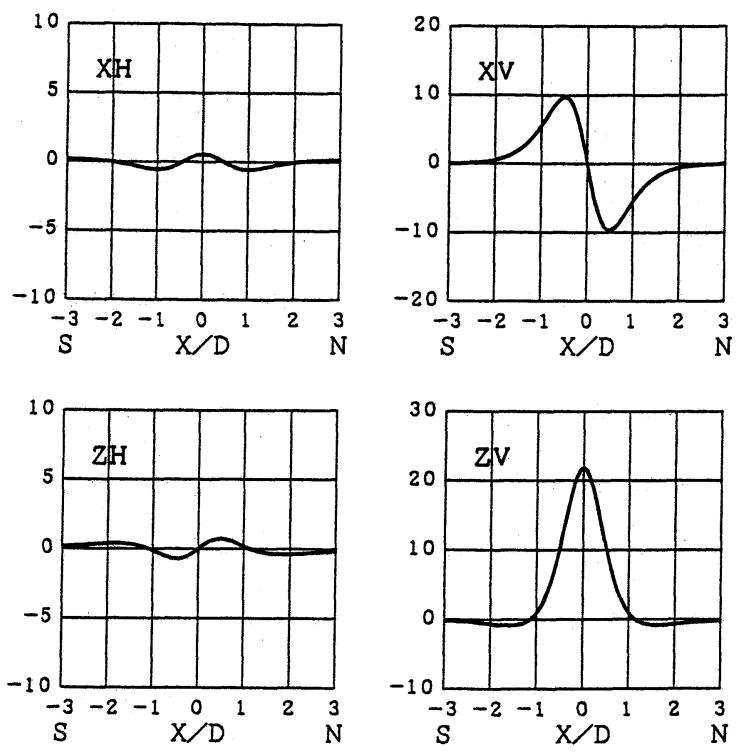

(a)
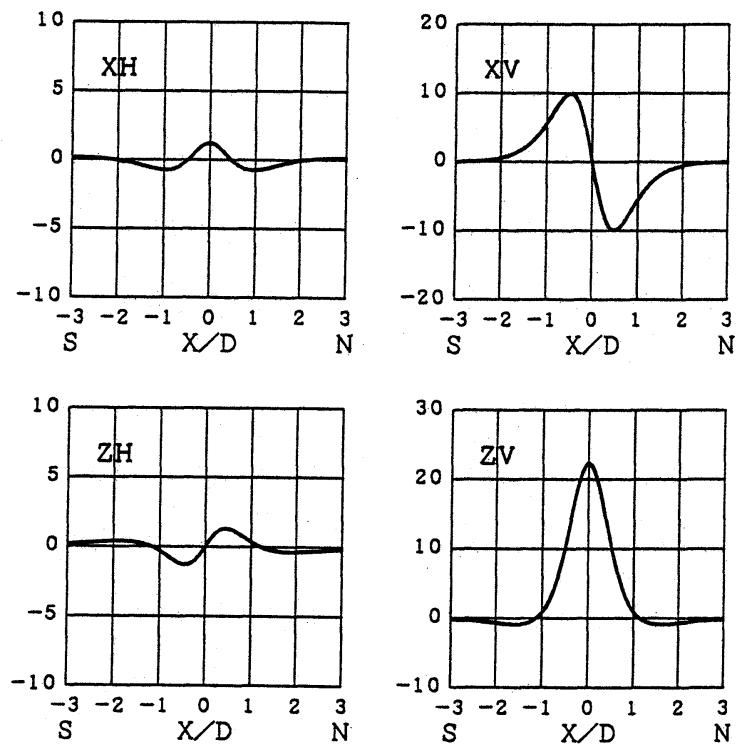

(b)

Fig. 9. Magnetic components along the N-S meridian in the case of $H=D(=12 \mathrm{~km})$ for (a) $b=1 \mathrm{~km}$, (b) $b=300 \mathrm{~m}$, (c) $b=30 \mathrm{~m}$. 

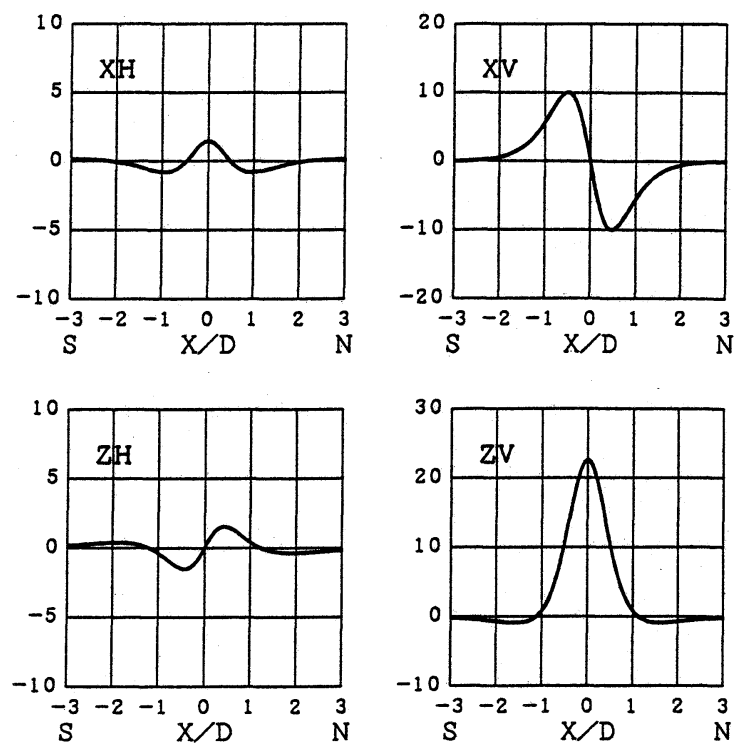

(c)

Fig. 9. (continued).

\subsection{Surface distribution of the magnetic field on the Earth}

Finally, we present the surface distribution. Figure 10 shows the total intensity changes as we may expect from the Kilauea Volcano following Davis' model parameters in Table 1. DAvis (1976) gave a numerical result for this model (i.e. Fig. 3, of his paper). Figure 10 exhibits a slightly different pattern from Davis' results. However, the positions and values of positive and negative peaks are roughly coincident with each other. Hence Davis' numerical computations are confirmed again by the present analytic approach.

Comparing Fig. 10 with Figs. 4(a) and 4(b), we recognize that the finite spherical source solution apparently resembles the type II solution of the point source problem. It is also noticeable that the finite source solution gives rather bigger changes than the point source one. This tendency is ascertained for larger values of $b / D$. Such a result is interesting because the stress-induced magnetization itself is weaker for a larger value of $b$. It is presumed that the source of the magnetic lines of force, i.e. the spherical surface of the demagnetized area consisting of single and double layers, comes closer to the observation site as $b$ increases. This can be regarded as a sort of the enhancement effect due to non-uniform magnetization in the Earth's crust (e.g. OSHIMAN, 1990).

Let us investigate the Curie depth dependency of the surface distribution. Coming back again to SUZUKI and OSHIMAN's (1990) parameters, we put $D=12 \mathrm{~km}, b=3 \mathrm{~km}$, $I_{0}=45^{\circ}$, and $D_{0}=0^{\circ}$. In Figs. 11,12 and 13 the total intensity changes for $H=5 \mathrm{~km}, 12 \mathrm{~km}$ and $20 \mathrm{~km}$, are respectively shown. The pattern of piezomagnetic changes is highly dependent on the spatial configuration of the demagnetized sphere and the Curie point isotherm. In particular, the sign of magnetic field changes turns over depending on whether or not the Curie depth $H$ lies deeper than the source depth $D$. 


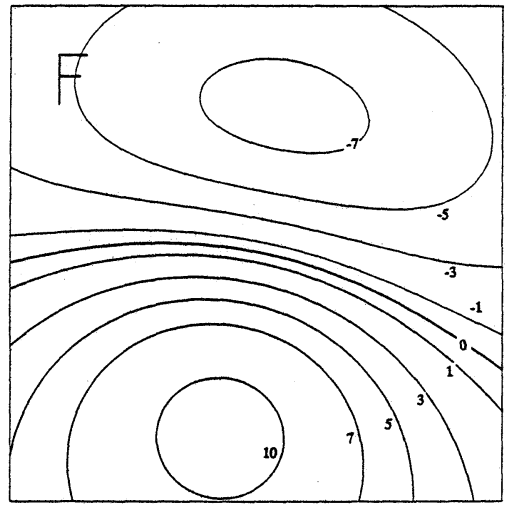

Fig. 10.

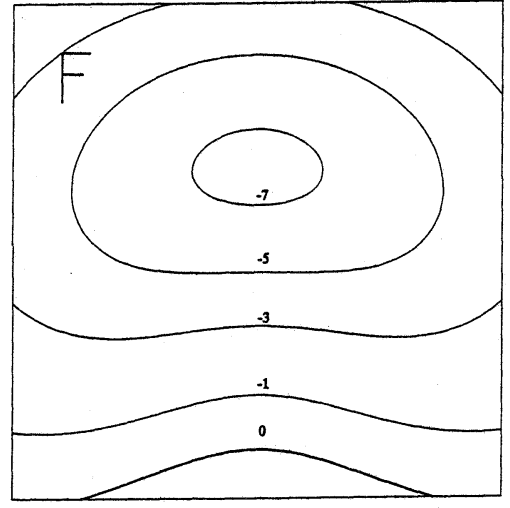

Fig. 11.

Fig. 10. Surface distribution of total intensity changes for Kilauea Volcano, with the same model parameters as Davis' (1976). Unit in nT.

Fig. 11. Total intensity changes for $\boldsymbol{H}=5 \mathrm{~km}$. Model parameters are SUZuKI and OshimAN's (1990). Unit in $\mathrm{nT}$.

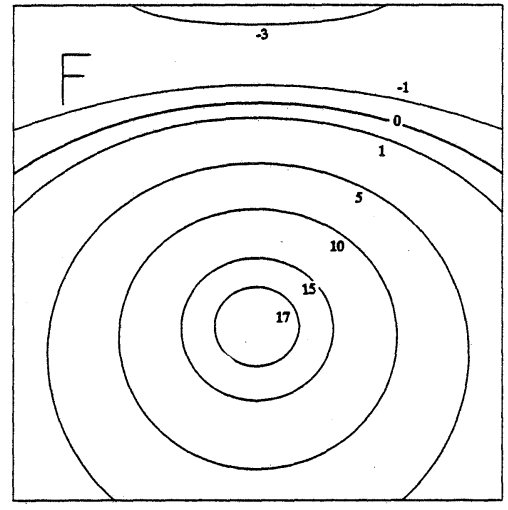

Fig. 12.

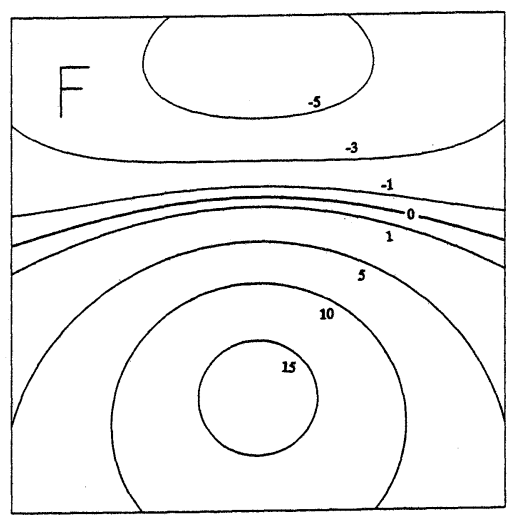

Fig. 13.

Fig. 12. Total intensity changes for $H=12 \mathrm{~km}$. Model parameters are SUZUKI and Oshiman's (1990). Unit in $\mathrm{nT}$.

Fig. 13. Total intensity changes for $H=20 \mathrm{~km}$. Model parameters are SUZUKı and OsHIMAN's (1990). Unit in $\mathrm{nT}$.

\section{Point Source Solution as a Limiting Case of Finite Spherical Source}

From the previous section, we find that the general feature of the finite source solution resembles to that of the type II solution as the source radius diminishes. We will demonstrate here that the type II solution is actually obtained by reducing the source radius $b$ to zero in the type III solution. As pointed out in Section 3 , the influence of the source sphere is abruptly added as the Curie depth $H$ crosses over the source depth $D$. Accordingly, the magnetic potential varies discontinuously at $H=D$. The transient 
behavior of the solution around $H=D$ will be presented by investigating the limiting case. The cause of the discrepancy between the type I and the type II solutions will be clarified. We find that the type II solution is appropriate for the point source solution: this issue comes to a conclusion.

Let us investigate the integrals over the spherical surface, i.e. Eq. (4.2). Among various terms in the integrand, those arising from $\boldsymbol{u}^{(\mathrm{B})}$ are finite at the source point $(0,0, D)$ and are multiplied by $b$. They vanish in the limit of diminishing $b$ to zero. We have only to take account of the contributions from $\boldsymbol{u}^{(\mathrm{A})}$ :

$$
\begin{aligned}
& \frac{2 \mu}{C} F_{1 x}^{(\mathrm{KA})}=\frac{2(3 \lambda+4 \mu)}{3 \lambda+2 \mu} \frac{\sin \theta}{b}, \\
& \frac{2 \mu}{C} F_{2 x}^{(\mathrm{KA})}=\sin \theta, \\
& \frac{2 \mu}{C} F_{1 z}^{(\mathrm{KA})}=\frac{2(3 \lambda+4 \mu)}{(3 \lambda+2 \mu)} \frac{\cos \theta}{b}, \\
& \frac{2 \mu}{C} F_{2 z}^{(\mathrm{KA})}=\cos \theta .
\end{aligned}
$$

According to Appendix B, the unit potential functions, $G_{1 x}$ etc., have power series representations of $k^{2}$. Expanding them further into a Tailor series of $b$, we obtain the following:

$$
\begin{aligned}
& G_{1 x}=\frac{\pi}{4} r_{0} \cos \phi_{0} \frac{b}{\rho_{1}^{3}} \sin \theta\{1+O(b)\}, \\
& G_{2 x}=-2 \pi r_{0} \cos \phi_{0} \sin \frac{\theta}{\rho_{1}^{3}}\{1+O(b)\}, \\
& G_{1 z}=2 \pi\left\{\frac{1}{\rho_{1}}+\frac{b \zeta_{0} \cos \theta}{\rho_{1}^{3}}+O\left(b^{2}\right)\right\}, \\
& G_{2 z}=-2 \pi \zeta_{0} \frac{\cos \theta}{\rho_{1}^{3}}\{1+O(b)\} .
\end{aligned}
$$

Substituting Eqs. (6.1) and Eqs. (6.2) into Eq. (4.2) and integrating by terms, we have

$$
\begin{aligned}
\frac{2 \pi}{C} W_{x}^{(\mathrm{KA})}= & C_{x} \frac{3 \pi(\lambda+2 \mu)}{3 \lambda+2 \mu} \frac{x_{0}}{\rho_{1}^{3}} \int_{\Theta}^{\pi} \sin ^{3} \theta \mathrm{d} \theta+O(b) \\
\frac{2 \pi}{C} W_{z}^{(\mathrm{KA})}= & C_{z} \frac{4 \pi(3 \lambda+4 \mu)}{3 \lambda+2 \mu} \frac{1}{b \rho_{1}} \int_{\Theta}^{\pi} \sin \theta \cos \theta \mathrm{d} \theta \\
& +C_{z} \frac{6 \pi(\lambda+2 \mu)}{3 \lambda+2 \mu} \frac{\zeta_{0}}{\rho_{1}^{3}} \int_{\Theta}^{\pi} \cos ^{2} \theta \sin \theta \mathrm{d} \theta+O(b) .
\end{aligned}
$$

In case that $H>D$, the infinitesimal source sphere is fully included within the 
magnetized region: we may put $\Theta=0$. The first term on the right-hand side of Eq. (6.3b) becomes null. Taking a limit of $b$ approaching zero, we obtain

$$
\begin{aligned}
& \frac{2 \mu}{C} W_{x}^{(\mathrm{KA})}=C_{x} \frac{4 \pi(\lambda+2 \mu)}{3 \lambda+2 \mu} \frac{x_{0}}{\rho_{1}^{3}}, \\
& \frac{2 \pi}{C} W_{z}^{(\mathrm{KA})}=C_{z} \frac{4 \pi(\lambda+2 \mu)}{3 \lambda+2 \mu} \frac{-D_{1}}{\rho_{1}^{3}}
\end{aligned}
$$

in which we use the relation $\zeta_{0}=z_{0}-D=-D_{1}$ (cf. Eq. (4.8)). Equations (6.4) are coincident with Eqs. (3.18). As it was reasonable to expect, the limit of the type III solution as diminishing the source radius $b$ becomes the type II solution.

Next we will investigate a special case in which $H=D$. Since the source surface is a hemisphere, we may put $\Theta=\pi / 2$. From Eqs. (6.3) we obtain

$$
\begin{aligned}
& \frac{2 \pi}{C} W_{x}^{(\mathrm{KA})}=C_{x} \frac{2 \pi(\lambda+2 \mu)}{3 \lambda+2 \mu} \frac{x_{0}}{\rho_{1}^{3}}, \\
& \frac{2 \pi}{C} W_{z}^{(\mathrm{KA})}=-C_{z} \frac{2 \pi(3 \lambda+4 \mu)}{3 \lambda+2 \mu} \frac{1}{b \rho_{1}}+C_{z} \frac{2 \pi(\lambda+2 \mu)}{3 \lambda+2 \mu} \frac{-D_{1}}{\rho_{1}^{3}} .
\end{aligned}
$$

The first term on the right-hand side of Eq. (6.5b) diverges as we reduce $b$ to zero. However, we will find immediately after that a counterpart of this divergent term emerges in the Curie surface integral and completely cancel with each other. Then, only the second term in Eq. (6.5b) survives. Notice that the integrals over the hemispherical surface are just one half of those over the entire spherical surface.

We are now to evaluate the Curie surface integral for $H=D . f_{1 k}^{(\mathrm{B})}$ and $f_{2 k}^{(\mathrm{B})}$ have no singularity at $(0,0, D)$. The integral containing them is reduced to elementary functions: i.e. terms having $\rho_{3}$ in Eqs. (3.3). We should examine integrals involving $f_{1 k}^{(\mathrm{A})}$ and $f_{2 k}^{(\mathrm{A})}$. Putting $H=D$ in Eqs. (4.19a), (4.19c), (4.19e) and (4.19g), we have

$$
\begin{aligned}
& \frac{2 \mu}{C} f_{1 x}^{(\mathrm{A})}=0, \\
& \frac{2 \mu}{C} f_{2 x}^{(\mathrm{A})}=\frac{1}{u^{2}}, \\
& \frac{2 \mu}{C} f_{1 z}^{(\mathrm{A})}=\frac{3 \lambda+4 \mu}{3 \lambda+2 \mu} \frac{1}{u^{3}}, \\
& \frac{2 \mu}{C} f_{2 z}^{(\mathrm{A})}=0 .
\end{aligned}
$$

The lower limit of integration $u_{0}$ is equal to $b$. We denote the Curie surface integral contributed from $\boldsymbol{u}^{(\mathrm{A})}$ with a superscript (HA). We now seek to obtain the limit of the following integral as we let $b$ approach zero: 


$$
\begin{aligned}
& \frac{2 \mu}{C} W_{x}^{(\mathrm{HA})}=C_{x} 2\left(z_{0}-D\right) \cos \phi_{0} \int_{b}^{\infty} \frac{1}{u} \Phi_{4} \mathrm{~d} u, \\
& \frac{2 \mu}{C} W_{z}^{(\mathrm{HA})}=C_{z} \frac{2(3 \lambda+4 \mu)}{3 \lambda+2 \mu} \int_{b}^{\infty} \frac{1}{u^{2}} \Phi_{1} \mathrm{~d} u .
\end{aligned}
$$

Referring to Appendix A, we find

$$
\begin{aligned}
& \Phi_{4}=\frac{\pi}{D_{1}} \int_{0}^{\infty} J_{1}(u t) J_{1}\left(r_{0} t\right) \mathrm{e}^{-D_{1} t} t \mathrm{~d} t \\
& \Phi_{1}=\pi \int_{0}^{\infty} J_{0}(u t) J_{0}\left(r_{0} t\right) \mathrm{e}^{-D_{1} t} \mathrm{~d} t
\end{aligned}
$$

in which we use the relation $\zeta=H-z_{0}=D_{1}$. Substituting Eqs. (6.8) into Eqs. (6.7) and interchanging the order of integrations with respect to $t$ and $u$, we have

$$
\begin{aligned}
& \frac{2 \mu}{C} W_{x}^{(\mathrm{HA})}=-2 \pi C_{x} \cos \phi_{0}\left\{\lim _{b \rightarrow 0} \int_{0}^{\infty} I_{x}(b) J_{1}\left(r_{0} t\right) \mathrm{e}^{-D_{1} t} t \mathrm{~d} t\right\}, \\
& \frac{2 \mu}{C} W_{z}^{(\mathrm{HA})}=-2 \pi C_{z} \frac{3 \lambda+4 \mu}{3 \lambda+2 \mu}\left\{\lim _{b \rightarrow 0} \int_{0}^{\infty} I_{z}(b) J_{0}\left(r_{0} t\right) \mathrm{e}^{-D_{1} t} \mathrm{~d} t\right\}
\end{aligned}
$$

where

$$
\begin{aligned}
& I_{x}(b)=\int_{b}^{\infty} \frac{J_{1}(u t)}{u} \mathrm{~d} u, \\
& I_{z}(b)=\int_{b}^{\infty} \frac{J_{0}(u t)}{u^{2}} \mathrm{~d} u .
\end{aligned}
$$

With the aid of the reccurrence formula, we obtain

$$
I_{x}(b)=t I_{0}(b)+J_{1}(b t)
$$

where

$$
I_{0}(b)=\int_{b}^{\infty} J_{0}(u t) \mathrm{d} u
$$

Since $I_{0}(0)=1 / t$ and hence $I_{x}(0)=1$, we can evaluate Eq. (6.9a) as follows:

$$
\begin{aligned}
\frac{2 \mu}{C} W_{x}^{(\mathrm{HA})} & =-2 \pi C_{x} \cos \phi_{0} \int_{0}^{\infty} J_{1}\left(r_{0} t\right) \mathrm{e}^{-D_{1} t} t \mathrm{~d} t \\
& =-2 \pi C_{x} \cos \phi_{0} \frac{r_{0}}{\left(r_{0}^{2}+D_{1}^{2}\right)^{3 / 2}}=-2 \pi C_{x} \frac{x_{0}}{\rho_{1}^{3}} .
\end{aligned}
$$


The integral $I_{z}(b)$ is, however, divergent if $b=0$. Taking account of a power series expansion of Bessel function $J_{0}$, we find that $\left\{J_{0}(u t)-1\right\}$ is $O\left(u^{2}\right)$. Thus an integral $\int_{0}^{\infty}\left(\left(J_{0}(u t)-1\right) / u^{2}\right) \mathrm{d} u$ is convergent. We may rewrite $I_{z}(b)$ as follows:

$$
I_{z}(b)=\int_{b}^{\infty} \frac{J_{0}(u t)-1}{u^{2}} \mathrm{~d} u+\int_{b}^{\infty} \frac{1}{u^{2}} \mathrm{~d} u=I_{1}(b)+\frac{1}{b}
$$

where

$$
I_{1}(b)=\frac{J_{0}(b t)-1}{b}-t J_{1}(b t)-t^{2} I_{0}(b)
$$

Since $I_{1}(0)=-t$, we have

$$
\int_{0}^{\infty} I_{1}(0) J_{0}\left(r_{0} t\right) \mathrm{e}^{-D_{1} t} \mathrm{~d} t=-\int_{0}^{\infty} J_{0}\left(r_{0} t\right) \mathrm{e}^{-D_{1} t} t \mathrm{~d} t=-\frac{D_{1}}{\rho_{1}^{3}}
$$

Thus we may evaluate the integral $(6.9 \mathrm{~b})$ for a small value of $b$ as

$$
\frac{2 \mu}{C} W_{z}^{(\mathrm{HA})}=2 \pi C_{z} \frac{3 \lambda+4 \mu}{3 \lambda+2 \mu}\left\{-\frac{D_{1}}{\rho_{1}^{3}}+O(b)+\frac{1}{b \rho_{1}}\right\} .
$$

Comparing Eq. (6.16) with Eq. (6.5b), we find that the divergent term in $W_{z}^{(\mathrm{KA})}$ is completely cancelled out by the divergent one in $W_{z}^{(\mathrm{HA})}$. Since we always consider the total sum $W_{z}^{(\mathrm{KA})}+W_{z}^{(\mathrm{HA})}$, the divergent term disappears.

Thus we can separate a finite part $I_{1}(0)$ from a divergent integral $I_{z}(0)$. It is called Hadamard's "finite part ( partie finie) of divergent integral", which is properly incorporated in the framework of the distribution theory (SCHWARTZ, 1966). According to the theory of distributions or hyperfunctions (IMAI, 1981), the finite part or the pf integral is defined as

$$
\begin{aligned}
\mathrm{pf} \int_{0}^{a} & \frac{1}{x^{m}} \phi(x) \mathrm{d} x \\
= & -\left\{\frac{1}{m-1} \frac{\phi(a)}{a^{m-1}}+\frac{1}{(m-1)(m-2)} \frac{\phi^{\prime}(a)}{a^{m-2}}+\cdots+\frac{1}{(m-1) !} \frac{\phi^{(m-2)}(a)}{a}\right\} \\
& +\frac{1}{(m-1) !}\left\{\phi^{(m-1)}(a) \log a+\left(1+\frac{1}{2}+\cdots+\frac{1}{m-1}\right) \phi^{(m-1)}(0)\right. \\
& \left.-\int_{0}^{a} \phi^{(m)}(x) \log x \mathrm{~d} x\right\}
\end{aligned}
$$

where $m$ is a positive integer and $\phi(x)$ is any analytic function regular on the $x$ axis. Actually $I_{1}(0)$ can be calculated by putting $m=2$ and $\phi(x)=J_{0}(x t)$ in the formula (6.17).

Taking account of Eq. (6.4), Eq. (6.13), and Eq. (6.16), we should rewrite the type II solution in Eqs. (3.19) as follows: 


$$
\begin{aligned}
& \frac{2 \mu}{C} W_{x}^{(\mathrm{II})}=4 \pi C_{x}\left[\frac{\mu}{3 \lambda+2 \mu}\left(\frac{x_{0}}{\rho_{1}^{3}}-\frac{x_{0}}{\rho_{3}^{3}}\right)+\frac{6(\lambda+\mu)}{3 \lambda+2 \mu} H \frac{3 x_{0} D_{3}}{\rho_{3}^{5}}\right.
\end{aligned}
$$

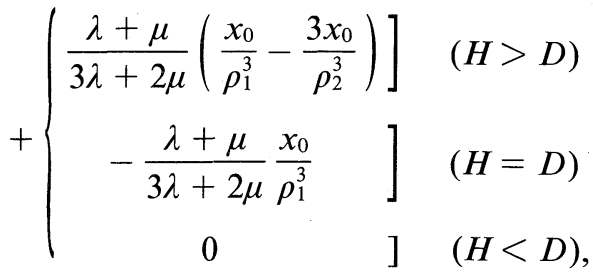

$$
\begin{aligned}
& \frac{2 \mu}{C} W_{z}^{(\mathrm{II})}=4 \pi C_{z}\left[-\frac{\mu}{3 \lambda+2 \mu}\left(\frac{D_{1}}{\rho_{1}^{3}}-\frac{D_{3}}{\rho_{3}^{3}}\right)+\frac{6(\lambda+\mu)}{3 \lambda+2 \mu} H\left(-\frac{1}{\rho_{3}^{3}}+\frac{3 D_{3}^{2}}{\rho_{3}^{5}}\right)\right.
\end{aligned}
$$

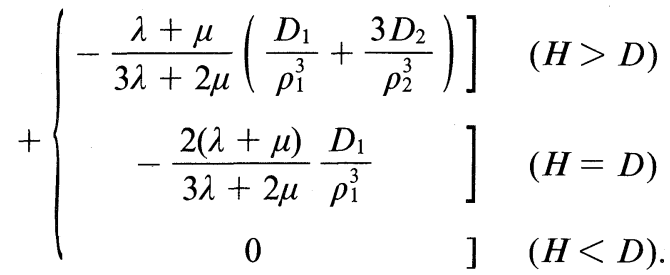

The magnetic potential has a gap across $H=D$. The potential value at $H=D$ is just the average of the two limiting values for $H=D-0$ and $H=D+0$.

Figure 14 shows the Curie depth dependency of two magnetic components at the origin for (a) type I and (b) type II solution, respectively. The discontinuity at $H=D$ in the type II solution can be considered as an extreme of steep increase in the magnetic field

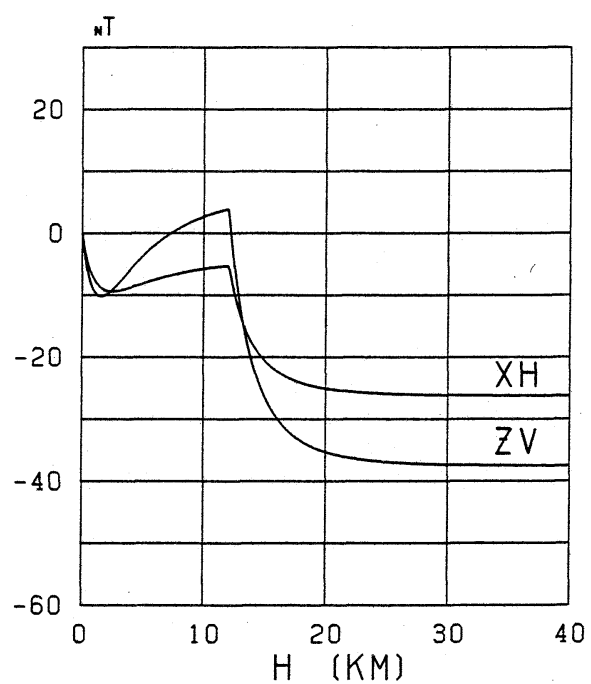

(a)

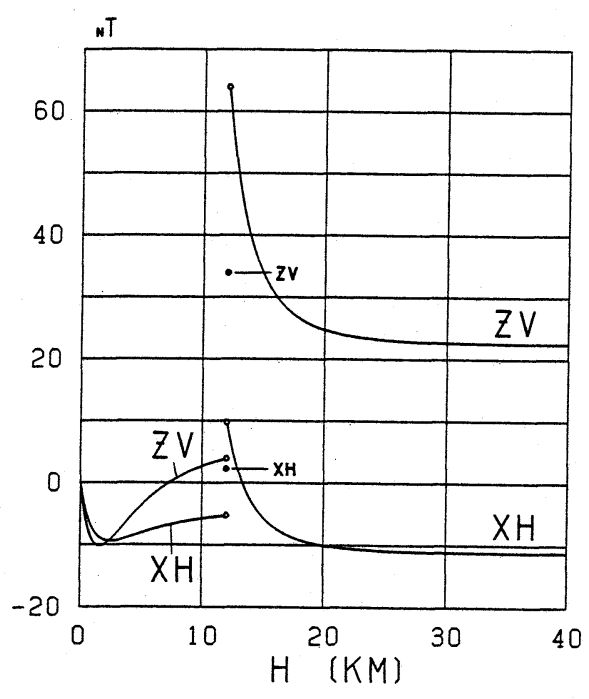

(b)

Fig. 14. Curie depth dependency of the two magnetic components at the origin for the point source solution of (a) type I, and (b) type II respectively. Model parameters are SUZUKI and OsHiman's (1990). 
around $H=D$ for a small value of $b$ as shown in Fig. 7(b). The nature of the discrepancy between the two types of solutions is obvious: the type I solution is the remainder after subtracting the effect of the source sphere from the type II solution. Comparing Eqs. (3.7) and Eqs. (6.18), we find the difference between type II and type I as follows:

$$
\begin{gathered}
\frac{2 \mu}{C} W_{x}^{(\mathrm{II})}-\frac{2 \mu}{C} W_{x}^{(\mathrm{I})}=4 \pi C_{x} \times\left\{\begin{array}{cc}
-\frac{2(\lambda+\mu)}{3 \lambda+2 \mu} \frac{x_{0}}{\rho_{1}^{3}} & (H>D) \\
-\frac{\lambda+\mu}{3 \lambda+2 \mu} \frac{x_{0}}{\rho_{1}^{3}} & (H=D) \\
0 & (H<D),
\end{array}\right. \\
\frac{2 \mu}{C} W_{z}^{(\mathrm{II})}-\frac{2 \mu}{C} W_{z}^{(\mathrm{I})}=4 \pi C_{z} \times\left\{\begin{array}{cc}
-\frac{4(\lambda+\mu)}{3 \lambda+2 \mu} \frac{D_{1}}{\rho_{1}^{3}} & (H>D) \\
-\frac{2(\lambda+\mu)}{3 \lambda+2 \mu} \frac{D_{1}}{\rho_{1}^{3}} & (H=D) \\
0 & (H<D) .
\end{array}\right.
\end{gathered}
$$

As we have already seen in the derivation process of the type II solution from the type III one, "the effect of the source sphere" comes from the sources of magnetic lines of force along the spherical surface. These surface sources originate entirely from the magnetized region excluding the pressure source. Hence we should naturally suspect if we overlook any portion of piezomagnetic components produced within the whole magnetic body in the type I case.

Now let us recall the definition for the integration area of the two kinds of solutions as depicted in Fig. 2. According to the arguments in Section 3, the magnetic potential $W_{k}^{(\mathrm{I})}$ for type I and $W_{k}^{(\mathrm{II})}$ for type II are given as the sum of a few surface potentials:

$$
\begin{aligned}
& W_{k}^{(\mathrm{I})}=W_{k}^{(\mathrm{O})}+W_{k}^{(\mathrm{P})}+W_{k}^{(\mathrm{H})}, \\
& W_{k}^{(\mathrm{II})}=W_{k}^{(\mathrm{O})}+W_{k}^{(\mathrm{K})}+W_{k}^{(\mathrm{H})}
\end{aligned}
$$

where

$$
W_{k}^{(\mathrm{P})}=W_{k}^{(H=D-\varepsilon)}-W_{k}^{(H=D+\varepsilon)} .
$$

Before taking a limit of $\varepsilon$ to zero, we find that a disagreement with respect to the integration area exists between the two. It is a region surrounded by two horizontal planes $H=D-\varepsilon$ and $H=D+\varepsilon$ and the source sphere as shown in Fig. 15. We specify this area with $K^{\prime}$. The contribution of $K^{\prime}$ to the potential is given by

$$
W_{k}^{\left(\mathrm{K}^{\prime}\right)}=W_{k}^{(\mathrm{K})}-W_{k}^{(H=D-\varepsilon)}+W_{k}^{(H=D+\varepsilon)} .
$$

In the limit of $\varepsilon$ approaching zero, the volume of the region $K^{\prime}$ becomes null. In evaluating the type I solution, we had implicitly taken it for granted, that the contribution from $K^{\prime}$ vanishes as well. This is incorrect: the region $K^{\prime}$ reduces to a thin 


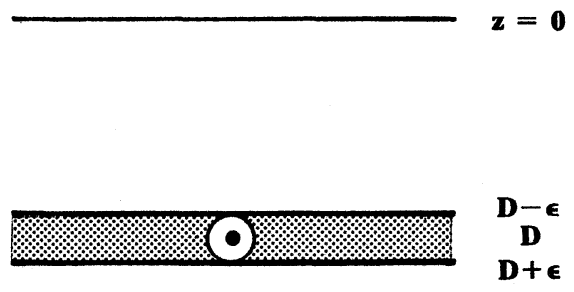

Fig. 15. A schematic representation of the integration area $K^{\prime}$ (hatched), which is ignored in the type I solution.

layer at $H=D$ but produces a finite amount of magnetic field. The relationship among these surface potentials as given by Eqs. (6.20) through (6.23) holds good even for the limiting case of $\varepsilon \rightarrow 0$. Then we have

$$
W_{k}^{(\mathrm{I})}+W_{k}^{\left(\mathrm{K}^{\prime}\right)}=W_{k}^{(\mathrm{II})} .
$$

In other words, the jump in the potential values arises from the stress-induced magnetization in a thin layer at $H=D$.

All these considerations imply that the type II solution properly evaluates the contributions from the whole piezomagnetic constituents. Hence we arrive at a final conclusion: the type II solution is correct, as that for the point source problem. The earlier result by SASAI (1979), i.e. the type I solution, should be rejected. This conclusion compels serious alterations for some of the earlier works of Sasai. It is because the same way of integration around singular points has been undertaken in constructing Green's functions for tectonomagnetic modeling (i.e. SASAI, 1980, 1986) that I am now reexamining all these previous results (SASAI, 1990).

SASAI (1979) derived the type I solution under the direct suggestion of HAGIWARA's (1977) work on the gravity change associated with the Mogi model. Hagiwara conducted the integration to obtain the density-related gravity change in quite the same way as the type I solution. A question arises: is Hagiwara's solution for gravity change erroneous? Since $\boldsymbol{u}^{(\mathrm{A})}=C / 2 \mu \nabla\left(R_{1}^{-1}\right)$, the density change at an arbitrary point due to $\boldsymbol{u}^{(\mathrm{A})}$ is given by

$$
\Delta \rho=\rho \operatorname{div} \boldsymbol{u}^{(\mathrm{A})}=\rho \frac{C}{2 \mu} \nabla^{2}\left(R_{1}^{-1}\right)=-4 \pi \rho \frac{C}{2 \mu} \delta\left(\boldsymbol{R}_{1}\right) .
$$

Hagiwara put the term zero, because $\nabla^{2}\left(R_{1}^{-1}\right)=0$ everywhere except $R_{1}=0$. According to the characteristics of Dirac's delta function, the density integral due to $\boldsymbol{u}^{(\mathrm{A})}$ produces a point mass at $(0,0, D)$. Instead, he took into account the loss of surrounding mass due to inflation of the source sphere. Recently OKUBO (1990) formulated the gravity changes due to point dislocations. He demonstrated that the gravity produced by the delta function is nothing but the mass loss due to the inflation of the pressure source. Consequently, HAGIWARA's (1977) result for the gravity change due to the Mogi model is correct. 
In our calculations the delta function does not appear explicitly. However, the treatment of the problem in the form of surface integrals is verified with the aid of Green's theorem. Actually the equivalence of $\nabla^{2}\left(R_{1}^{-1}\right)$ to $-4 \pi \delta\left(\boldsymbol{R}_{1}\right)$ is also verified through Green's theorem, so that our derivation of $W_{k}^{(\mathrm{K})}$ is just the same procedure as we apply the delta function. In evaluating the potential value for $H=D$ in the type II solution, we require of a mathematical operation corresponding to "half" of the delta function. In the one-dimensional theory of distributions, we meet an operator called $\delta_{ \pm}$ function: i.e.

$$
\delta_{ \pm}=\frac{1}{x \pm i \varepsilon}=P \frac{1}{x} \mp i \pi \delta(x)
$$

where $P$ implies Cauchy's principal value. The procedure in which we obtain the point source solution for $H=D$ is nothing but a 3-dimensional version of $\delta_{ \pm}$function.

The physical interpretation of the source term in the gravity change by OKUBO (1990) does not hold as it is in the case of piezomagnetism. In the Mogi model the source radius expands by $\Delta a=a \Delta P / 4 \mu$ under an internal pressure $\Delta P$. The magnetic potential corresponding to the disappearance of the magnetized spherical shell of radius $a$ and thickness $\Delta a$ is given by

$$
\begin{aligned}
& W_{x}^{(\Delta a)}=m_{x} \frac{x_{0}}{\rho_{1}^{3}}, \\
& W_{z}^{(\Delta a)}=m_{z} \frac{z_{0}-D}{\rho_{1}^{3}}
\end{aligned}
$$

where

$$
m_{k}=4 \pi a^{2} \Delta a J_{k}=2 \pi \frac{C}{\mu} J_{k}
$$

Comparing Eqs. (6.25) with Eqs. (6.19), we find

$$
W_{k}^{(\mathrm{K})}=\frac{1}{2} \frac{\lambda+2 \mu}{\lambda+\mu} \beta \mu W_{k}^{(\Delta a)} .
$$

The source term due to the type II solution is amplified by $(1 / 2)((\lambda+2 \mu) /(\lambda+\mu)) \beta \mu$ times the source expansion effect. This factor amounts to 26.25 for $\beta=1.0 \times 10^{-4} \mathrm{bar}^{-1}$ and $\lambda=\mu=3.5 \times 10^{5}$ bar. This amplification originates from the fundamental nature of piezomagnetism caused by the quantum mechanical spin-spin interactions. As it has been discussed by SASAI (1985), the piezomagnetic effect is predominant by an order of magnitude or more as compared with other causes of magnetic change due to mechanical distortion, i.e. free-air effect, the field produced by an upheaved portion of the earth's surface, and the source expansion effect. This relationship is true even if we adopt the type II solution instead of the type I.

The question raised by SUZUKI and OSHIMAN (1990) should also be reexamined: 
they showed that the type I solution, which turned out to be incorrect, could be reproduced even by numerical 3-D integrations. It is beyond the scope of the present paper to discuss the numerical 3-D integration procedure. We may only say that the way to compute the contribution from the $K^{\prime}$ region would play a key role. In numerical 3-D integrations, the effect of mesh size has so far been our principal concern. However, the present problem indicates that the arrangement of volume elements around a singular point is also of crucial importance.

As for the application to volcanology, the type III solution provides us with an effective tool to calculate the volcanomagnetic effect. Even the type II solution consisting of elementary functions works as a good approximation for $b / D$ less than 0.3 or so. Recent observations brought to light a variety of volcanomagnetic effects: e.g. JOHNSTON et al. (1981), ZLOTNICKI (1986), ZLOTNICKI et al. (1986), YUKUTAKE et al. (1990a, b), SASAI et al. (1990), HAMANO et al. (1990). In the Kilauea Volcano, the coeruptive magnetic change was much smaller than that anticipated from the Mogi model (DAVIS et al., 1979). In view of this fact, DAVIS (1986) went further to propose a new model for magma reservoir, which is made up of an ellipsoidal inclusion in an elastic half-space. His "pseudo chamber" model is versatile enough to interpret actual surface deformations on Kilauea Volcano. The present study should give us suggestions for computing the piezomagnetic changes associated with Davis' pseudo chamber model.

I am greatly indebted to Yoshiko Suzuki and Naoto Oshiman whose work motivated the present study. Discussions on the various aspects of the problem with Naoto Oshiman and Shuhei Okubo were very helpful. My hearty thanks are also due to Paul Davis, Yoshikazu Tanaka, Hisashi Utada and Yasunori Nishida for their suggestions and encouragement. Malcolm Johnston and two anonymous reviewers critically read the manuscript to give me useful comments and suggestions for the revision, which I acknowledge sincerely.

\section{Appendix A: Reduction of $\Phi$ Integrals to Lipschitz-Hankel Type Ones}

The integral of Lipschitz-Hankel type is defined by EASON et al. (1955) as

$$
I(\mu, v ; \lambda)=\int_{0}^{\infty} J_{\mu}(a t) J_{v}(b t) \mathrm{e}^{-c t} t^{\lambda} \mathrm{d} t \quad(c>0) .
$$

We consider here only the case that $\lambda, \mu$ and $v$ are integers, and hence we replace these letters with $l, m$ and $n$. The problem is to reduce the following integral into the form of Eq. (A.1).

$$
\Phi=\int_{0}^{\pi} \frac{\cos (j \psi)}{\left\{\rho_{\psi}\right\}^{k}} \mathrm{~d} \psi
$$

in which $j$ and $k$ are non-negative integers. $\rho_{\psi}$ is given by

$$
\rho_{\psi}=\sqrt{R^{2}+c^{2}} \quad(c>0)
$$

where 


$$
R^{2}=a^{2}-2 a b \cos \psi+b^{2}
$$

Such a simple form of integral cannot be expressed with elementary functions. This sort of integrals inevitably appear in any potential problems with axial symmetry.

Let us first investigate

$$
\Phi_{1}=\int_{0}^{\pi} \frac{1}{\rho_{\psi}} \mathrm{d} \psi
$$

We start from Lipschitz integral (WATSON, 1922):

$$
\int_{0}^{\infty} \mathrm{e}^{-c t} J_{0}(R t) \mathrm{d} t=\frac{1}{\sqrt{R^{2}+c^{2}}} .
$$

Substituting Eq. (A.6) into Eq. (A.5) and changing the order of integrations with respect to $t$ and $\psi$, we obtain

$$
\Phi_{1}=\int_{0}^{\infty} \mathrm{e}^{-c t} \mathrm{~d} t \int_{0}^{\pi} J_{0}(R t) \mathrm{d} \psi
$$

We recall Gegenbauer's addition theorem (WATSON, 1922):

$$
J_{0}(R t)=J_{0}(a t) J_{0}(b t)+2 \sum_{m=1}^{\infty} J_{m}(a t) J_{m}(b t) \cdot \cos m \psi
$$

Integrating both sides of Eq. (A.8) with respect to $\psi$ from 0 to $\pi$, we find

$$
\int_{0}^{\pi} J_{0}(R t) \mathrm{d} \psi=\pi J_{0}(a t) J_{0}(b t)
$$

which is put into (A.7) to obtain

$$
\Phi_{1}=\pi \int_{0}^{\infty} J_{0}(a t) J_{0}(b t) \mathrm{e}^{-c t} \mathrm{~d} t=\pi I(0,0 ; 0) .
$$

Differentiating (A.5) with respect to $c$ and taking Eq. (A.10) into account, we find

$$
\Phi_{3}=-\frac{1}{c} \frac{\partial \Phi_{1}}{\partial c}=\frac{\pi}{c} I(0,0 ; 1)
$$

The procedure to obtain Eq. (A.10) and Eq. (A.11) is the fundamental technique to reduce $\Phi$ functions to Lipschitz-Hankel type integrals. We summarize only the results by the following equations:

$$
\begin{aligned}
& \Phi_{2}=\pi I(1,1 ; 0), \\
& \Phi_{4}=\frac{\pi}{c} I(1,1 ; 1),
\end{aligned}
$$




$$
\begin{aligned}
& \Phi_{5}=\frac{\pi}{c} I(2,2 ; 1), \\
& \Phi_{6}=\frac{\pi}{3 c^{3}}\{c I(0,0 ; 2)+I(0,0 ; 1)\}, \\
& \Phi_{7}=\frac{\pi}{3 c^{3}}\{c I(1,1 ; 2)+I(1,1 ; 1)\}, \\
& \Phi_{8}=\frac{\pi}{3 c^{3}}\{c I(2,2 ; 2)+I(2,2 ; 1)\}, \\
& \Phi_{9}=\frac{\pi}{3 c^{3}}\{c I(3,3 ; 2)+I(3,3 ; 1)\} .
\end{aligned}
$$

The reason why we use a more complicated expression of the Lipschitz-Hankel type is obvious: differentials of these functions (A.1) with respect to $a, b$, and $c$ reduce again to the same type of integrals with different values of $m, n$ or $l$. In Appendix B we will find that these functions are expressible with complete elliptic integrals. To directly differentiate complete elliptic integrals is, however, a mathematical mess.

It is worth mentioning that RIKITAKE (1951) presented an analytic expression for the magnetic field produced by a uniformly magnetized circular cone. His formula consists of complete elliptic integrals, the derivation of which is based essentially on the same procedure as described here.

Appendix B: The Integrals of Lipschitz-Hankel Type as Expressed by Complete Elliptic Integrals

Some of the integrals (A.1) have already been given as combinations of complete elliptic integrals by EASON et al. (1955). We follow their notations. They define complete elliptic integrals of the first and the second kind multiplied by $2 / \pi$ as follows.

$$
\begin{aligned}
& F_{0}(k)=\frac{2}{\pi} \int_{0}^{\pi / 2} \frac{\mathrm{d} \psi}{\sqrt{1-k^{2} \sin ^{2} \psi}} \\
& E_{0}(k)=\frac{2}{\pi} \int_{0}^{\pi / 2} \sqrt{1-k^{2} \sin ^{2} \psi} \mathrm{d} \psi
\end{aligned}
$$

where

$$
k^{2}=\frac{4 a b}{(a+b)^{2}+c^{2}} \text {. }
$$

$a, b$, and $c$ are parameters in the integral (A.1). We need $I(0,0 ; 0), I(1,1 ; 0), I(0,0 ; 1)$, $I(1,1 ; 1), I(0,0 ; 2), I(1,1 ; 2), I(2,2 ; 1), I(2,2 ; 2), I(3,3 ; 1)$, and $I(3,3 ; 2)$. The first four functions are found in EASON et al.'s (1955) table, and are reproduced here: 


$$
\begin{aligned}
& I(0,0 ; 0)=\frac{k}{2 \sqrt{a b}} F_{0}(k), \\
& I(1,1 ; 0)=\frac{1}{k \sqrt{a b}}\left\{\left(1-\frac{1}{2} k^{2}\right) F_{0}(k)-E_{0}(k)\right\}, \\
& I(0,0 ; 1)=\frac{c k^{3}}{8 k^{\prime 2}(a b)^{3 / 2}} E_{0}(k), \\
& I(1,1 ; 1)=\frac{c k}{4(a b)^{3 / 2}}\left\{\left(1-\frac{1}{2} k^{2}\right) k^{\prime-2} E_{0}(k)-F_{0}(k)\right\}
\end{aligned}
$$

where $k^{\prime 2}=1-k^{2}$.

The rest of the higher order functions are reducible to linear combinations of low order (i.e. $m, n=0$ or $1, l=1,0$ or -1 ) with the aid of the recurrence relationship. In that case, however, we should refer to $I(1,1 ;-1), I(1,0 ; 0)$ and so on, which require of Heumann's lambda function or of the complete elliptic integral of the third kind. Instead we will derive the expressions for higher order functions directly.

This can be easily achieved because the remaining six functions have the form of either $I(n, n ; 1)$, or $I(n, n ; 2)$. EASON et al. (1955) gave a formula:

$$
I(n, n ; 1)=\frac{(-1)^{n} c k^{3}}{4 \pi(a b)^{3 / 2}} \int_{0}^{\pi / 2} \frac{\cos (2 n \psi) \mathrm{d} \psi}{\left(1-k^{2} \sin ^{2} \psi\right)^{3 / 2}}
$$

Differentiating (B.8) with respect to $c$, we have

$$
\begin{aligned}
I(n, n ; 2)= & -\frac{\partial}{\partial c} I(n, n ; 1) \\
= & \frac{(-1)^{n} k^{3}}{4 \pi(a b)^{3 / 2}}\left\{-\int_{0}^{\pi / 2} \frac{\cos 2 n \psi}{\left(1-k^{2} \sin ^{2} \psi\right)^{3 / 2}} \mathrm{~d} \psi\right. \\
& \left.\quad+\frac{3 c^{2} k^{2}}{4 a b} \int_{0}^{\pi / 2} \frac{\cos 2 n \psi}{\left(1-k^{2} \sin ^{2} \psi\right)^{5 / 2}} \mathrm{~d} \psi\right\}
\end{aligned}
$$

Some additional formulas are necessary for further calculations:

$$
\begin{aligned}
& \int_{0}^{\pi / 2} \frac{\mathrm{d} \psi}{\left(1-k^{2} \sin ^{2} \psi\right)^{3 / 2}}=\frac{1}{k^{\prime 2}} E(k), \\
& \int_{0}^{\pi / 2} \frac{\mathrm{d} \psi}{\left(1-k^{2} \sin ^{2} \psi\right)^{5 / 2}}=-\frac{1}{3 k^{\prime 2}} F(k)+\frac{2\left(k^{\prime 2}+1\right)}{3 k^{\prime 4}} E(k), \\
& \int_{0}^{\pi / 2}\left(1-k^{2} \sin ^{2} \psi\right)^{3 / 2} \mathrm{~d} \psi=\frac{2}{3}\left(1+k^{\prime 2}\right) E(k)-\frac{k^{\prime 2}}{3} F(k) .
\end{aligned}
$$

(Equation (B.11) is correspondent to Eq. (3.7) in EASON et al. (1955). The latter is incorrect, probably owing to a misprint.) 
Putting $n=0$ through 3 in Eqs. (B.8) and (B.9), we can obtain the following:

$$
\begin{aligned}
& I(0,0 ; 2)=\frac{k^{3}}{8(a b)^{3 / 2} k^{\prime 2}}\left[-E_{0}(k)\right. \\
& \left.+\frac{c^{2} k^{2}}{4 a b}\left\{-F_{0}(k)+\frac{2\left(1+k^{\prime 2}\right)}{k^{\prime 2}} E_{0}(k)\right\}\right], \\
& I(1,1 ; 2)=\frac{k}{8(a b)^{3 / 2} k^{\prime 2}}\left[2{k^{\prime}}^{2} F_{0}(k)-\left(1+k^{\prime 2}\right) E_{0}(k)\right. \\
& \left.-\frac{c^{2} k^{2}}{4 a b}\left\{\left(1+k^{\prime 2}\right) F_{0}(k)-\frac{2\left(1-k^{2}+k^{4}\right)}{k^{\prime 2}} E_{0}(k)\right\}\right], \\
& I(2,2 ; 1)=\frac{c}{8(a b)^{3 / 2} k}\left\{-8\left(2-k^{2}\right) F_{0}(k)+\frac{k^{4}-16 k^{2}+16}{k^{\prime 2}} E_{0}(k)\right\}, \\
& I(2,2 ; 2)=\frac{1}{8(a b)^{3 / 2} k}\left[8\left(2-k^{2}\right) F_{0}(k)-\frac{k^{4}-16 k^{2}+16}{k^{\prime 2}} E_{0}(k)\right. \\
& +\frac{c^{2} k^{2}}{4 a b k^{\prime 2}}\left\{\left(16-16 k^{2}-k^{4}\right) F_{0}(k)\right. \\
& \left.\left.-\frac{2\left(2-k^{2}\right)\left(4-4 k^{2}-k^{4}\right)}{k^{\prime 2}} E_{0}(k)\right\}\right] \text {, } \\
& I(3,3 ; 1)=\frac{c}{8(a b)^{3 / 2} k^{3}}\left[\left(2-k^{2}\right)\left\{\frac{k^{4}-16 k^{2}+16}{k^{\prime 2}}+\frac{80}{3}\right\} E_{0}(k)\right. \\
& \left.-\left\{6\left(3 k^{4}-16 k^{2}+16\right)-\frac{32}{3} k^{\prime 2}\right\} F_{0}(k)\right], \\
& I(3,3 ; 2)=\frac{1}{8(a b)^{3 / 2} k^{3}}\left[-\left(2-k^{2}\right)\left\{\frac{k^{4}-16 k^{2}+16}{k^{\prime 2}}+\frac{80}{3}\right\} E_{0}(k)\right. \\
& +\left\{6\left(3 k^{4}-16 k^{2}+16\right)-\frac{32}{3} k^{\prime 2}\right\} F_{0}(k) \\
& -\frac{3 c^{2} k^{2}}{4 a b k^{\prime 2}}\left\{2 \left(9 k^{4}-64 k^{2}+64\right.\right. \\
& \left.-\frac{\left(k^{2}-2\right)^{2}\left(k^{4}-16 k^{2}+16\right)}{3 k^{\prime 2}}\right) E_{0}(k) \\
& \left.\left.+\left(k^{2}-2\right)\left(48 k^{\prime 2}-\frac{k^{4}-16 k^{2}+16}{3}\right) F_{0}(k)\right\}\right] .
\end{aligned}
$$

All these functions have $a b$ and $k$ in the denominators, which cause numerical instability when $a$ or $b$ approaches zero. For small values of $a, b$, and $k$, we have power series expansions with respect to $k$, with the aid of series representations for $F_{0}(k)$ and 
$E_{0}(k)$. They are given as follows:

$$
\begin{aligned}
& I(0,0 ; 0)=\frac{1}{\sqrt{(a+b)^{2}+c^{2}}}\left\{1+\frac{1}{4} k^{2}+\frac{9}{64} k^{4}+\cdots\right\} \\
& I(1,1 ; 0)=\frac{1}{8} \frac{k^{2}}{\sqrt{(a+b)^{2}+c^{2}}}\left\{1+\frac{3}{4} k^{2}+\frac{75}{128} k^{4}+\cdots\right\} \\
& I(0,0 ; 1)=\frac{c}{\left\{(a+b)^{2}+c^{2}\right\}^{3 / 2}}\left\{1+\frac{3}{4} k^{2}+\frac{45}{64} k^{4}+\cdots\right\} \\
& I(1,1 ; 1)=\frac{3}{8} \frac{c k^{2}}{\left\{(a+b)^{2}+c^{2}\right\}^{3 / 2}}\left\{1+\frac{5}{4} k^{2}+\frac{175}{128} k^{4}+\cdots\right\} \\
& I(0,0 ; 2)=\frac{1}{\left\{(a+b)^{2}+c^{2}\right\}^{3 / 2}}\left[-\left\{1+\frac{3}{4} k^{2}+\frac{45}{64} k^{4}+\cdots\right\}\right. \\
& \left.\quad+\frac{3 c^{2}}{(a+b)^{2}+c^{2}}\left\{1+\frac{5}{4} k^{2}+\frac{105}{64} k^{4}+\cdots\right\}\right]
\end{aligned}
$$

$$
\begin{aligned}
I(1,1 ; 2)= & \frac{3}{8} \frac{k^{2}}{\left\{(a+b)^{2}+c^{2}\right\}^{3 / 2}} \\
& \times\left[-\left\{1+\frac{5}{4} k^{2}+\frac{175}{128} k^{4}+\cdots\right\}\right. \\
& \left.+\frac{5 c^{2}}{(a+b)^{2}+c^{2}}\left\{1+\frac{7}{4} k^{2}+\frac{315}{128} k^{4}+\cdots\right\}\right],
\end{aligned}
$$

$$
\begin{aligned}
& I(2,2 ; 1)=\frac{15}{128} \frac{c k^{4}}{\left\{(a+b)^{2}+c^{2}\right\}^{3 / 2}}\left\{1+\frac{7}{4} k^{2}+\cdots\right\} \\
& I(2,2 ; 2)=\frac{15}{128} \frac{k^{4}}{\left\{(a+b)^{2}+c^{2}\right\}^{3 / 2}}\left[-\left\{1+\frac{7}{4} k^{2}+\cdots\right\}\right.
\end{aligned}
$$

$$
\left.+\frac{7 c^{2}}{(a+b)^{2}+c^{2}}\left\{1+\frac{9}{4} k^{2}+\cdots\right\}\right]
$$

$$
I(3,3 ; 1)=\frac{35}{1024} \frac{c k^{6}}{\left\{(a+b)^{2}+c^{2}\right\}^{3 / 2}}\left\{1+\frac{9}{4} k^{2}+\cdots\right\},
$$$$
I(3,3 ; 2)=\frac{35}{1024} \frac{k^{6}}{\left\{(a+b)^{2}+c^{2}\right\}^{3 / 2}}\left[-\left\{1+\frac{9}{4} k^{2}+\cdots\right\}\right.
$$

$$
\left.+\frac{9 c^{2}}{(a+b)^{2}+c^{2}}\left\{1+\frac{11}{4} k^{2}+\cdots\right\}\right] \text {. }
$$

$\Phi_{6}, \Phi_{7}, \Phi_{8}$ and $\Phi_{9}$ are of a form: 


$$
\begin{aligned}
\Phi[n] & =\frac{\pi}{3 c^{3}}\{c I(n, n ; 2)+I(n, n ; 1)\} \\
& =\frac{(-1)^{n} k^{5}}{16(a b)^{5 / 2}} \int_{0}^{\pi / 2} \frac{\cos 2 n \psi}{\left(1-k^{2} \sin ^{2} \psi\right)^{5 / 2}} \mathrm{~d} \psi
\end{aligned}
$$

Hence these functions have simpler expressions, which are easily obtained through Eqs. (B.14) to (B.18).

\section{Appendix C: Integral over Curie Surface with No Hole}

In Eq. (4.18) we have to integrate the following functions with respect to $u$ from 0 to infinity: i.e. $u^{2} \Phi_{2} / S^{5}, u^{2} \Phi_{2} / S^{7}, u^{2} \Phi_{4} / S^{3}, u^{2} \Phi_{4} / S^{5}, u^{2} \Phi_{4} / S^{7}, u \Phi_{1} / S^{3}, u \Phi_{1} / S^{5}, u \Phi_{3} / S^{3}$, $u \Phi_{3} / S^{5}$, and $u \Phi_{3} / S^{7}$. Here we put

$$
S=\sqrt{u^{2}+\zeta_{1}^{2}}\left(\zeta_{1}>0\right), \quad \zeta_{1}=|H-D| \quad \text { or } \quad H+D .
$$

$\Phi_{1}$ through $\Phi_{4}$ are represented by Lipschitz-Hankel type integrals, in which only $J_{1}(u t)$ or $J_{0}(u t)$ are involved as a function of $u$. Interchanging the order of integrations with respect to $t$ and $u$, we first perform integrations with respect to $u$. For example,

$$
\begin{aligned}
\int_{0}^{\infty} \frac{u^{2}}{S^{5}} \Phi_{2} \mathrm{~d} u & =\pi \int_{0}^{\infty} \frac{u^{2}}{S^{5}} \mathrm{~d} u \int_{0}^{\infty} J_{1}(u t) J_{1}\left(r_{0} t\right) \mathrm{e}^{-\zeta_{1} t} \mathrm{~d} t \\
& =\pi \int_{0}^{\infty}\left[\int_{0}^{\infty} \frac{J_{1}(u t)}{S^{5}} u^{2} \mathrm{~d} u\right] J_{1}\left(r_{0} t\right) \mathrm{e}^{-\zeta_{1} t} \mathrm{~d} t
\end{aligned}
$$

We need the following six integrals:

$$
\begin{aligned}
& I_{1}=\int_{0}^{\infty} \frac{u^{2}}{S^{3}} J_{1}(u t) \mathrm{d} u, \quad I_{2}=\int_{0}^{\infty} \frac{u^{2}}{S^{5}} J_{1}(u t) \mathrm{d} u, \quad I_{3}=\int_{0}^{\infty} \frac{u^{2}}{S^{7}} J_{1}(u t) \mathrm{d} u, \\
& I_{4}=\int_{0}^{\infty} \frac{u}{S^{3}} J_{0}(u t) \mathrm{d} u, \quad I_{5}=\int_{0}^{\infty} \frac{u}{S^{5}} J_{0}(u t) \mathrm{d} u, \quad I_{6}=\int_{0}^{\infty} \frac{u}{S^{7}} J_{0}(u t) \mathrm{d} u .
\end{aligned}
$$

$I_{n}$ 's $(n=1, \ldots, 6)$ are obtained by differentiating the following function $I_{0}$ with respect to $\zeta_{1}$ or $t$ under the integral sign:

$$
I_{0}=\int_{0}^{\infty} \frac{1}{\sqrt{u^{2}+\zeta_{1}^{2}}} J_{0}(u t) u \mathrm{~d} u=\frac{\mathrm{e}^{-\zeta_{1} t}}{t}
$$

This can be evaluated by applying Hankel's inversion theorem to the Lipschitz integral, i.e. Eq. (A.6). Then we obtain the following:

$$
I_{4}=-\frac{1}{\zeta_{1}} \frac{\partial I_{0}}{\partial \zeta_{1}}=\frac{1}{\zeta_{1}} \mathrm{e}^{-\zeta_{1} t}, \quad I_{1}=-\frac{\partial I_{4}}{\partial t}=\mathrm{e}^{-\zeta_{1} t}
$$




$$
\begin{array}{ll}
I_{5}=-\frac{1}{3 \zeta_{1}} \frac{\partial I_{4}}{\partial \zeta_{1}}=\frac{1+\zeta_{1} t}{3 \zeta_{1}^{3}} \mathrm{e}^{-\zeta_{1} t}, & I_{2}=-\frac{\partial I_{5}}{\partial t}=\frac{t}{3 \zeta_{1}} \mathrm{e}^{-\zeta_{1} t} \\
I_{6}=-\frac{1}{5 \zeta_{1}} \frac{\partial I_{5}}{\partial \zeta_{1}}=\frac{3+3 \zeta_{1} t+\zeta_{1}^{2} t^{2}}{15 \zeta_{1}^{5}} \mathrm{e}^{-\zeta_{1} t}, & I_{3}=-\frac{\partial I_{6}}{\partial t}=\frac{t+\zeta_{1} t^{2}}{15 \zeta_{1}^{3}} \mathrm{e}^{-\zeta_{1} t}
\end{array}
$$

The next step is integration with respect to $t$. This can be easily done: e.g.

$$
\int_{0}^{\infty} I_{1} J_{1}\left(r_{0} t\right) \mathrm{e}^{-\zeta t} t \mathrm{~d} t=\int_{0}^{\infty} J_{1}\left(r_{0} t\right) \mathrm{e}^{-\left(\zeta+\zeta_{1}\right) t} t \mathrm{~d} t=\frac{r_{0}}{\left\{r_{0}^{2}+\left(\zeta+\zeta_{1}\right)^{2}\right\}^{3 / 2}}
$$

We conduct integrations for all the combinations involving $I_{n}$ 's in Eq. (4.18). Thus we have

$$
\begin{aligned}
\frac{2 \mu}{C} W_{x}^{(\mathrm{H})}=2 \pi C_{x}[ & -\frac{3 \lambda+4 \mu}{3 \lambda+2 \mu} \operatorname{sgn}(H-D) \frac{x_{0}}{\rho_{12}^{3}} \\
& +\left\{\left(\frac{\lambda+3 \mu}{\lambda+\mu}-\frac{2 \mu}{3 \lambda+2 \mu}\right) \frac{x_{0}}{\rho_{3}^{3}}+\frac{6(3 \lambda+4 \mu)}{3 \lambda+2 \mu} H D_{3} \frac{x}{\rho_{3}^{5}}\right\} \\
& \left.-\frac{x_{0}}{\rho_{12}^{3}}+\left\{-\frac{\lambda+3 \mu}{\lambda+\mu} \frac{x_{0}}{\rho_{3}^{3}}+6 H D_{3} \frac{x_{0}}{\rho_{3}^{5}}\right\}\right] \\
\frac{2 \mu}{C} W_{z}^{(\mathrm{H})}=2 \pi C_{z}[ & -\frac{3 \lambda+4 \mu}{3 \lambda+2 \mu} \frac{H-z_{0}+|H-D|}{\rho_{12}^{3}} \\
& +\left\{-\left(\frac{3 \lambda+4 \mu}{3 \lambda+2 \mu} \cdot \frac{\lambda-\mu}{\lambda+\mu}+\frac{8 \mu}{3 \lambda+2 \mu}\right) \frac{D_{3}}{\rho_{3}^{3}}\right. \\
& \left.+2 H \frac{3 \lambda+4 \mu}{3 \lambda+2 \mu}\left(-\frac{1}{\rho_{3}^{3}}+\frac{3 D_{3}^{2}}{\rho_{3}^{5}}\right)\right\} \\
& -\operatorname{sgn}(H-D) \frac{H-z_{0}+|H-D|}{\rho_{12}^{3}} \\
& \left.+\left\{\frac{\lambda+3 \mu}{\lambda+\mu} \frac{D_{3}}{\rho_{3}^{3}}+2 H\left(-\frac{1}{\rho_{3}^{3}}+\frac{3 D_{3}^{2}}{\rho_{3}^{5}}\right)\right\}\right]
\end{aligned}
$$

where

$$
\rho_{12}=\sqrt{r_{0}^{2}+\left(H-z_{0}+|H-D|\right)^{2}} .
$$

It can readily be ascertained that Eqs. (C.5) coincide with Eqs. (3.3).

Looking back through the derivation process, we realize that we made the same operation as the Fourier transform and its inverse. The double Fourier transform is reduced to the Hankel transform in the cylindrical coordinates, which is nothing but calculating $I_{n}$ 's. Then we integrated with respect to $t$, which can be regarded as a wave number. This operation corresponds to the inverse Fourier transformation. If we go further as to modify the Lipschitz-Hankel type integrals to complete elliptic ones, we 
encounter complicated expressions with respect to $u$. Hence it is too difficult to integrate such functions.

\section{REFERENCES}

DAVIS, P. M., The computed piezomagnetic anomaly field for Kilauea volcano, Hawaii, J. Geomag. Geoelectr., 28, 113-122, 1976.

Davis, P. M., Surface deformation due to inflation of an arbitrarily oriented triaxial ellipsoidal cavity in an elastic half-space, with reference to Kilauea Volcano, Hawaii, J. Geophys. Res., 91, 7429-7438, 1986.

Davis, P. M., F. D. StaCeY, C. J. Zablocki, and J. V. Olson, Improved signal discrimination in tectonomagnetism: Discovery of a volcanomagnetic effect at Kilauea, Hawaii, Phys. Earth Planet. Inter., 19, 331-336, 1979.

EAson, G., B. NOBLE, and I. N. SNeddon, On certain integrals of Lipschitz-Hankel type involving products of Bessel functions, Phil. Trans. R. Soc., London, Ser. A, 247, 529-551, 1955.

Fiske, R. S. and W. T. KinoshitA, Inflation of Kilauea Volcano prior to its 1967-1968 eruption, Science, 165, 341-349, 1969.

Hagiwara, Y., The Mogi model as a possible cause of the crustal uplift in the eastern part of Izu Peninsula and related gravity change, Bull. Earthq. Res. Inst., Univ. Tokyo, 52, 301-309, 1977 (in Japanese).

Hamano, Y., H. Utada, T. Shimomura, Y. Tanaka, Y. Sasai, I. Nakagawa, Y. Yokoyama, M. Ohno, T. Yoshino, S. Koyama, T. Yukutake, and H. Watanabe, Geomagnetic variations observed after the 1986 eruption of Izu-Oshima Volcano, J. Geomag. Geoelectr., 42, 319-335, 1990.

Hastings, C., Jr., Approximations for Digital Computers, 205 pp., Princeton, 1955.

IMAI, I., Applicable Theory of Hyperfunctions, Vol. I, 210 pp., Saiensu, 1981 (in Japanese).

Johnston, M. J. S., Local magnetic field observations and stress changes near a slip discontinuity on the San Andreas fault, J. Geomag. Geoelectr., 30, 511-522, 1978.

Johnston, M. J. S., R. J. Muller, and J. Dvorak, Volcanomagnetic observations during eruptions, May-August 1980, in the 1980 eruptions of Mount St. Helens, Washington, USGS professional paper 1250, 183-189, 1981.

Mindlin, R. D. and D. H. Cheng, Nuclei of strain in the semi-infinite solid, J. Appl. Phys., 21, 926-930, 1950.

MOGI, K., Relations between the eruptions of various volcanoes and the deformations of the ground surfaces around them, Bull. Earthq. Res. Inst., Univ. Tokyo, 36, 99-134, 1958.

Ohshiman, N., Local geomagnetic changes associated with fault activity, M. Sc. Thesis, 178 pp., Tokyo Institute of Technology, 1980.

OKubo, S., Potential and gravity changes raised by a point dislocation, submitted to Geophys. J. Int., 1990.

Oshiman, N., Enhancement of tectonomagnetic change due to non-uniform magnetization in the Earth's crust-two-dimensional case studies, J. Geomag. Geoelectr., 42, 607-619, 1990.

RIKITAKE, T., The distribution of magnetic dip in Ooshima (Oo-sima) Island and its change that accompanied the eruption of Volcano Mihara, 1950, Bull. Earthq. Res. Inst., Univ. Tokyo, 29, 161-181, 1951.

SASAI, Y., The piezomagnetic field associated with the Mogi model, Bull. Earthq. Res. Inst., Univ. Tokyo, 54, $1-29,1979$.

SASAI, Y., Application of the elasticity theory of dislocations to tectonomagnetic modelling, Bull. Earthq. Res. Inst., Univ. Tokyo, 55, 387-447, 1980.

SASAI, Y., A surface integral representation of the tectonomagnetic field based on the linear piezomagnetic effect, Bull. Earthq. Res. Inst., Univ. Tokyo, 58, 763-785, 1983.

SASAI, Y., Predominance of piezomagnetic effect in tectonomagnetic field of the Mogi model, J. Geomag. Geoelectr., 37, 159-167, 1985.

SASAI, Y., A Green's function for tectonomagnetic problems in an elastic half-space, J. Geomag. Geoelectr., 38, 949-969, 1986.

SASAI, Y., Tectonomagnetic modeling on the basis of the linear piezomagnetic effect, 1990 (in preparation).

Sasal, Y., T. Shimomura, Y. Hamano, H. Utada, T. Yoshino, S. Koyama, Y. Ishikawa, I. NaKagawa, Y. Yokoyama, M. Ohno, H. Watanabe, T. Yukutake, Y. Tanaka, T. Yamamoto, K. Nakaya, S. Tsunomura, F. Muromatsu, and R. Murakami, Volcanomagnetic effect observed during the 1986 eruption of Izu-Oshima Volcano, J. Geomag. Geoelectr., 42, 291-317, 1990.

Schwartz, L., Théorie des Distributions, Herman, Paris, 1966 (Japanese translation by R. Iwamura et al., 410 pp., Iwanami Shoten, 1971). 
Stacey, F. D., K. G. BARR, and G. R. Robson, The volcano-magnetic effect, Pageoph, 62, 96-104, 1965.

SUZUKI, Y. and N. OsHIMAN, A paradox in volcanomagnetism: Disagreement between analytical and numerical estimates of geomagnetic changes due to an underground pressure nucleus, J. Geomag. Geoelectr., 42, 1291-1308, 1990.

TAKAHASI, H. and M. MORI, Double exponential formulas for numerical integration, Publ. R. I. M. S., Kyoto Univ., 9, 721-741, 1974.

Watson, G. N., A Treatise on the Theory of Bessel Functions, 4th ed., 804 pp., Cambridge, 1922.

YamaKawa, N., On the strain produced in a semi-infinite elastic solid by an interior source of stress, J. Seismol. Soc. Japan, (ii), 8, 84-98, 1955 (in Japanese).

Yukutake, T., H. UtADA, T. Yoshino, E. Kimoto, K. OTANi, and T. Shimomura, Regional secular change in the geomagnetic field in the Oshima Island area during a tectonically active period, J. Geomag. Geoelectr., 42, 257-275, 1990a.

Yukutake, T., H. Utada, T. Yoshino, H. Watanabe, Y. Hamano, Y. Sasai, E. Kimoto, K. Otani, and T. SHImomura, Changes in the geomagnetic total intensity observed before the eruption of Oshima Volcano in 1986, J. Geomag. Geoelectr., 42, 277-290, $1990 \mathrm{~b}$.

Zlotnicki, J., Magnetic measurements on La Soufriere Volcano, Guadeloupe (Lesser Antilles), 1976-1984: A re-examination of the volcanomagnetic effects observed during the volcanic crisis of 1976-1977, J. Volcanol. Geotherm. Res., 30, 83-116, 1986.

Zlotnicki, J., R. Verhille, J. P. Viode, J. C. Delmond, G. Simon, and P. Guillement, Magnetic network on the Montagne Pelée Volcano (Martinique, Lesser Antilles): A trial to discriminate volcanomagnetic signal, J. Geomag. Geoelectr., 38, 151-164, 1986. 